\title{
Haag's Theorem and Its Implications for the Foundations of Quantum Field Theory
}

\author{
John Earman and Doreen Fraser \\ Dept. of History and Philosophy of Science \\ University of Pittsburgh \\ Pittsburgh, PA 15260
}

\begin{abstract}
Although the philosophical literature on the foundations of quantum field theory recognizes the importance of Haag's theorem, it does not provide a clear discussion of the meaning of this theorem. The goal of this paper is to make up for this deficit. In particular, it aims to set out the implications of Haag's theorem for scattering theory, the interaction picture, the use of non-Fock representations in describing interacting fields, and the choice among the plethora of the unitarily inequivalent representations of the canonical commutation relations for free and interacting fields.
\end{abstract}

\section{Introduction}

Haag's theorem on the representation of interactions in quantum field theory (QFT) was first stated fifty years ago. Its importance was immediately recognized by the community of mathematical physicists who were developing axiomatic approaches to QFT. The aim of this paper is to call attention to the fact that Haag's theorem has implications for a host of foundational and philosophical issues surrounding QFT and to begin the project of assessing its impact.

The philosophy of science literature that touches on Haag's theorem shows a tendency to either overstatement or vacillation (or both). Teller's An interpretative introduction to quantum field theory (1995) tells the reader that "According to something called Haag's theorem there appears to be no known consistent formalism within which interacting quantum field theory can be expressed" (p. 115). Barton (1963, p. 157) is quoted as stating that "no field theory exists which differs from that of a free field." What Barton actually says is that that consequence follows from eight enumerated assumptions, and he makes it clear that at least one of the assumptions must be rejected. 
Sklar's Theory and Truth (2000) echoes Teller's presentation with some vacillation added; Haag's theorem, Sklar writes, "seemed to show the theory $[\mathrm{QFT}]$ incapable of describing interactions" (p. 28). This uncertainty about what Haag's theorem shows is not untypical of the philosophical literature (see for example Huggett and Weingard (1994, p. 376)). Heathcote (1989) provides a brief but accurate statement of Haag's theorem; but we will disagree below with one of the morals Heathcote attempts to draw from the theorem regarding the status of Fock representations. Bain (2000) gives a nice discussion of how the LSZ formalism is able to side-step one of the implications of Haag's theorem, ${ }^{1}$ but this presentation lacks a clear statement of Haag's theorem and is not complemented with an appreciation of what lessons Haag's theorem teaches us about how relativistic QFT differs from ordinary non-relativistic QM.

Our take on the significance of Haag's theorem falls into three parts. In the first instance, Haag's theorem should not be thought of as posing a problem for QFT but rather as illustrating the physical relevance of a mathematical fact that separates QFT from ordinary non-relativistic quantum mechanics (QM). In the former but not the latter case there exist unitarily inequivalent representations of the canonical commutation relations (CCR). Two representations of the CCR $\left(\mathcal{H}_{1},\left\{O_{1}^{i}\right\}\right)$ and $\left(\mathcal{H}_{2},\left\{O_{2}^{i}\right\}\right)$ (where $\left\{O_{n}^{i}\right\}$ is the collection of operators appearing in the CCR) are unitarily equivalent if and only if there exists some unitary mapping $U$ from Hilbert space $\mathcal{H}_{1}$ to Hilbert space $\mathcal{H}_{2}$ such that for each operator $O_{1}^{j} \in\left\{O_{1}^{i}\right\}$ there exists an operator $O_{2}^{j}=U O_{1}^{j} U^{-1} \in\left\{O_{2}^{i}\right\}$. The importance of unitary equivalence is that it guarantees physical equivalence in the sense that both representations deliver the same expectation values for corresponding observables. The question, then, is how to regard the unitarily inequivalent representations of the CCR with which we are confronted in QFT. One response would be to regard only one representation (up to unitary equivalence) as physically significant and dismiss the rest as unphysical. However, Haag's theorem rules out this response by showing that the existence of these inequivalent representations is not "a matter of mathematical sophistication without relevance to field theory," as Haag (1955, p. 18) himself put it in the article in which what has come to be known as Haag's theorem was first stated. ${ }^{2}$

In the second instance, however, while Haag's theorem does not show that no quantum field theory exists which differs from a free field theory, it does pose problems for some of the techniques used in textbook physics for extracting physical predictions from the theory. For example, it is true 
that Haag's theorem implies that, if the "interaction picture" is consistent, it does not describe non-trivial interactions. Since the interaction picture is the basis of the standard approach to scattering theory contained in most QFT textbooks, this may help to explain why Haag's theorem has elicited the degree of alarm exhibited in the above quotations. The alarm can be assuaged by noting that there are methods for treating scattering in QFT that do not rely on the interaction picture and, thus, do not run afoul of Haag's theorem. There is, however, unfinished business in explaining why perturbation theory works as well as it does.

In the third instance, there are interesting issues surrounding Haag's theorem that have received inadequate attention in the philosophical literature. Extant philosophical discussions of QFT routinely assume the availability of Fock representations. But the physics literature is replete with claims to the effect that, as a consequence of Haag's theorem, interacting fields have to be described by non-Fock representations. Moreover, the philosophical literature has little to offer on the issue of how a choice is to be made among the plethora of unitarily inequivalent representations of the CCR whose physical relevance is made vivid by Haag's theorem.

These issues are explored in the second part of this paper. The first part of the paper is devoted to the exegesis of Haag's theorem and discussion of its history. Considering that the theorem was formulated half a century ago, this undertaking is not as straightforward as one might expect. In his 1967 Cargèse lecture "Introduction to some aspects of the relativistic dynamics of quantized fields," Arthur Wightman characterized the textbook literature on Haag's theorem as "execrable" (1967, p. 255). One of the examples that he cites is Schweber (1962), ${ }^{3}$ one of the most widely used quantum field theory texts in the 1960s and 1970s. This is particularly surprising since Schweber was a co-author of Wightman and Schweber (1955), which anticipated Haag's theorem (see Section 2 below). Another text that fits Wightman's description is Bjorken and Drell's Relativistic quantum fields (1965), the other widely used text of the period. In their chapter on perturbation theory the authors assume that the interacting field and the incoming free field are connected at each time $t$ by a unitary transformation $U(t)$. In a footnote they say that while the existence of such a transformation is "a theorem of ordinary quantum mechanics, the proof breaks down for systems with a nondenumerable number of degrees of freedom" (note 5, p. 175), and they refer to Haag (1955). The authors then continue: "Here we assume the existence of $U(t)$." But Haag's theorem says not just that the proof of the existence of $U(t)$ 
breaks down in relativistic quantum field theory (QFT) but that, on pain of identifying the interacting field with the free field, the said transformation does not exist!

The text by Roman (1969, Sec. 8.4) contains a clear presentation of a proof of Haag's theorem, but the discussion of the implications of the theorem is marred by some misstatements. In particular, there is the invocation of what is termed a "converse" to a theorem of von Neumann, stating that irreducible representations of the equal-time canonical commutation relations (ETCCR) are unitarily equivalent. There is a theorem by von Neumann and Stone to this effect-for the case of a finite number of degrees of freedom. But for an infinite number of degrees of freedom, the theorem breaks down, and it is precisely this breakdown that is involved in Haag's theorem.

More recent textbooks escape Wightman's charge, but they do so by ignoring or saying very little about Haag's theorem. The currently most widely used and cited texts are Peskin and Schroeder (1995) and Weinberg (1995, 1996), neither of which mentions Haag's theorem. This silence is also generally true of the textbooks of the 1980s and 1990s. Our (admittedly unsystematic) survey found only two exceptions. In an appendix at the end of $A n$ introduction to quantum field theory, Sterman (1993) reviews the formalism of the interaction picture and then notes that Haag's theorem shows that the assumed unitary transformation between the incoming and interacting fields "is not strictly speaking consistent with Poincaré invariance." The reader is then referred elsewhere for more discussion of "this fascinating point, which however, has not been shown to affect practical results" (p. 508). A fuller account is contained in Ticciati's (1999) Quantum field theory for mathematicians, which bills Haag's theorem as pinpointing a "serious formal weakness in applying the interaction picture to field theory" (p. 84). Practice shows that

the result of applying the interaction picture to field theory is a perturbation series that does not converge [as implied by Haag's theorem] but whose first few terms yield wonderfully accurate predictions. It appears then that the interaction picture provides a sound approach to perturbation theory but may have no nonperturbative validity. (p. 84)

But why a method based on demonstrably false premises yields such "wonderfully accurate predictions" is a mystery that cries out for a resolution. 
The one exception to an otherwise disappointing textbook literature is Barton (1963, Ch. 14). It supplies an accessible proof Haag's theorem along with a generally good discussion of the significance of the theorem. The mild quibble we have with the latter is that the reader is left with a choice of three "schools of thought on what to do next." The most "optimistic" school of thought, the one Barton himself prefers, is to abandon the assumption that unitarily inequivalent representations of the CCR do not have to be employed in relativistic QFT. But this is not an optional "school of thought" but a demonstrable necessity. ${ }^{4}$

The plan of our paper is as follows. A brief sketch of the history of Haag's theorem is given in Section 2. Haag's original proof is reviewed in Section 3, along with the Hall-Wightman version of the theorem. Section 4 is devoted to some clarifications of and qualifications to the theorem. Explanations for why the interaction picture and perturbation theory work, in spite of Haag's theorem, are explored in Section 5. Section 6 discusses differences in the formulations of scattering theory in QFT and ordinary QM that result from Haag's theorem. Section 7 examines the claim that Haag's theorem shows that non-Fock representations are required to describe interacting fields. Haag's theorem tells us that the choice among the unitarily inequivalent representations of the CCR depends on the dynamics. The question of whether the choice is fully determined by the dynamics is taken up in Section 8. Concluding remarks are given in Section 9. Some technical details of important concepts are provided in the Appendix.

\section{How Haag's theorem came to be}

In non-relativistic QM quantization involves first and foremost the choice of a suitable representation of the CCR ("the choice problem"). In their most familiar guise the CCR take the form

$$
\left[q_{j}, q_{k}\right]=0=\left[p_{j}, p_{k}\right], \quad\left[q_{j}, p_{k}\right]=i \delta_{j k}
$$

where $j, k=1,2, \ldots$ index the degrees of freedom. For a finite number $n$ of degrees of freedom the most familiar representation of the CCR is the Schrödinger representation, which is obtained by taking the Hilbert space to be $L_{\mathbb{C}}^{2}\left(\mathbb{R}^{n}\right)$, the space of all complex-valued, square-integrable functions on 
$\mathbb{R}^{n}$, and setting

$$
\begin{aligned}
& q_{j} f\left(x_{1}, \ldots\right)=x_{j} f\left(x_{1}, \ldots\right) \quad f \in L_{\mathbb{C}}^{2}\left(\mathbb{R}^{n}\right) \\
& p_{k} f\left(x_{1}, \ldots\right)=i \frac{\partial}{\partial x_{k}} f\left(x_{1}, \ldots\right)
\end{aligned}
$$

An obvious technical drawback of (1)-(2) is that the operators $q_{j}$ and $p_{k}$ are unbounded and, thus, the equations are not meaningful until the domains of definition of the operators is stated. In order to avoid this problem, mathematicians prefer to use the exponentiated Weyl form of the canonical commutation relations (WCCR)

$$
\begin{gathered}
U_{j}(r) U_{k}(s)-U_{k}(s) U_{j}(r)=0=V_{j}(r) V_{k}(s)-V_{k}(s) V_{j}(r) \\
U_{j}(r) V_{k}(s)=V_{k}(s) U_{j}(r) \exp \left(i r s \delta_{j k}\right), \quad r, s \in \mathbb{R},
\end{gathered}
$$

which arise if $U_{j}(r)=\exp \left(i r p_{j}\right)$ and $V_{k}(s)=\exp \left(i s q_{k}\right)$. If the the representation of (3) is in terms of unitary groups of operators strongly continuous in $r$ and $s$, then (by Stone's theorem) the generators of the groups are selfadjoint operators, and on a common dense domain these operators satisfy (1). ${ }^{5}$ The form (3) of the CCR also makes possible the powerful Stone-von Neumann theorem, which states that, for a finite number of degrees of freedom, irreducible and strongly continuous representations of the WCCR by unitary groups of operators are all unitarily equivalent; indeed, they are all equivalent to the (exponentiated) Schrödinger representation. For ordinary QM and more generally for the quantum mechanics of systems with a finite number of degrees of freedom, this theorem seems to solve the choice problem, at least if the representations are required to be irreducible and strongly continuous; for then the theorem shows that the choice among such representations is a matter of convenience since it has no measurable physical consequences. ${ }^{6}$

When the number of degrees of freedom is infinite-as in QFT-the Stonevon Neumann theorem does not hold, and an uncountable number of unitarily inequivalent representations of the WCCR are available. This fact may have been known as early as the 1930s by the few mathematical physicists who were concerned with such matters, but as far as we are aware unitarily inequivalent representations were not explored in depth until the early 1950s, first in a book by Friedrichs (1953) and then more systematically by Gårding and Wightman (1954). The first glimmerings that inequivalent 
representations were not simply playthings of mathematicians but are necessarily implicated in the description of interacting quantum fields came from the work of van Hove (1952). The van Hove model was designed to provide an idealized but computationally tractable treatment of the interaction of a neutral scalar field with recoilless nucleons. The divergences van Hove encountered in the perturbation calculations were blamed on the fact that the state vectors for the interaction model are "orthogonal" to the state vectors in the Fock space of the free field, the said "orthogonality" being the physicists' lingo for the mathematical concept of disjoint representations which, for the case of irreducible representations, is coextensive with the concept of unitarily inequivalent representations (see Appendix). ${ }^{7}$

To Wightman and Schweber (1955) goes the credit for recognizing and stating in precise terms the significance of van Hove's model. They were emphatic that unitarily inequivalent representations "are not pathological phenomena whose construction requires mathematical trickery ... but occur in the most elementary examples of field theory" (p. 824), and motivated by van Hove's model they gave a toy example to illustrate the moral that the choice of representation depends on the dynamics of the field. ${ }^{8}$ However, they did not attempt to state or prove a theorem to show how this moral flows from the general features that a treatment of interacting fields are expected to satisfy.

Haag received a preprint of the Wightman and Schweber (1955) paper, ${ }^{9}$ and he obviously saw the need for providing the missing theorem. A statement of such a theorem was given in the same year by Haag (1955) in two versions. The first version has a heuristic proof that appeals to the form of typical Hamiltonians for interacting fields, while the second version was intended to be more general and does not invoke the form of typical Hamiltonians. However, Haag's proof of this second version is opaque. Hall and Wightman (1957) opined in a footnote that "In the opinion of the present authors, Haag's proof is, at least in part, inconclusive ..." (note 10, p. 41). They then add that "It will not have escaped the discerning reader of Haag's paper that, while we have generalized his results, eliminated one of his assumptions (the asymptotic condition), completed his proofs, and sharpened his conclusions, the essential physical points are Haag's" (ibid.). This addendum may have been in part an exercise in diplomacy, but it is also an accurate assessment; for while Haag's proof was inconclusive, the essential insights were his. In any case, although Hall and Wightman are responsible for applying the label "Haag's theorem"-a label that has stuck-the version of 
this theorem most often quoted in the physics literature is due to them. Thus, the "Haag-Hall-Wightman (HHW) theorem" would be a more appropriate label, and it will be applied here.

\section{The heuristic version of Haag's theorem and the Haag-Hall-Wightman theorem}

Before presenting the HHW theorem, it will be useful to state the gist of the heuristic version of Haag's original theorem (Haag 1955, pp. 30-1) which pops up again and again in the literature (see, for example, Wightman 1967). This argument takes the form of a reductio. Suppose that we are trying to describe both a free scalar field and a self-interacting scalar field using the same Hilbert space $\mathcal{H}$. Suppose that we demand of the vacuum state that it be the unique (up to phase) normalized state $|0\rangle \in \mathcal{H}$ that is invariant under Euclidean translations. And suppose that the vacuum state is the ground state in that it is an eigenstate of the Hamiltonian with eigenvalue $0 .{ }^{10}$ These suppositions are fulfilled in the case of the free scalar field with mass $m>0$, the usual no-particle state $\left|0_{F}\right\rangle$ ("bare vacuum"), and the free field Hamiltonian $H_{F}$. Since the vacuum state $\left|0_{I}\right\rangle$ of the interacting field ("dressed vacuum" or "physical vacuum") should also be invariant under Euclidean translations, it follows from the stated assumptions that $\left|0_{I}\right\rangle=c\left|0_{F}\right\rangle,|c|=1$, and since $\left|0_{I}\right\rangle$ is annihilated by the Hamiltonian $H$ for the interacting field, it follows that $H\left|0_{F}\right\rangle=0$. But the typical Hamiltonians for interacting fields take the form $H_{F}+H_{I}$, where $H_{I}$ describes the interaction of the field with itself, and such Hamiltonians do not annihilate $\left|0_{F}\right\rangle$ ( $H$ "polarizes the vacuum"). (As an illustration, consider the $\phi^{4}$ field $^{11}$ in $(3+1)$-dim Minkowski spacetime with (formal) Hamiltonian

$$
\begin{gathered}
H=H_{F}+\lambda \int: \phi^{4}: d^{3} x-C \\
\text { where } H_{F}=\frac{1}{2} \int\left\{m^{2}: \phi^{2}:+:|\nabla \phi|^{2}:+: \pi^{2}:\right\} d^{3} x
\end{gathered}
$$

and where $\pi$ is the momentum field conjugate to $\phi, C$ is a $c$-number constant chosen to give the ground state energy zero, and : : indicates the Wick normal product. This $H$ will not annihilate the bare vacuum $\left|0_{F}\right\rangle$ since the factor that follows the coupling constant $\lambda$ contains a term with a product 
of four creation operators (and there is only one term of this form, so it will not be canceled by another term) (Bogoliubov et al. 1975, Sec. 25.1)). Thus, we arrive at a contradiction: it follows from the announced assumptions that $H\left|0_{F}\right\rangle=0$, but for typical interaction Hamiltonians $H\left|0_{F}\right\rangle \neq 0$.

The relative simplicity of this first version of Haag's original theorem is purchased at the expense of generality since it appeals to the fact that typical Hamiltonians for interacting fields do not annihilate $\left|0_{F}\right\rangle$. It was the ambition of the second version of his theorem to dispense with any reference to the particular form of the interacting field Hamiltonian. However, it was Haag's attempted proof of this second version that Wightman found "inconclusive." A pair of theorems presented in Hall and Wightman (1957) was designed to fill the gap. We will label them the Haag-Hall-Wightman (HHW) theorem, Parts I and II; Hall and Wightman label them the first and second parts of the "Generalized Haag's theorem" because they apply to any pair of neutral scalar fields.

HHW Theorem, Part I. Consider two neutral scalar fields ${ }^{12} \phi_{j}, j=1,2$, and their conjugate momentum fields $\pi_{j}$ such that each pair $\left(\phi_{j}, \pi_{j}\right)$ gives an irreducible representation of the equal time CCR (ETCCR)

$$
\begin{aligned}
& {\left[\phi_{j}(\mathbf{x}, t), \pi_{j}\left(\mathbf{x}^{\prime}, t\right)\right]=i \delta\left(\mathbf{x}-\mathbf{x}^{\prime}\right) \quad j=1,2} \\
& {\left[\phi_{j}(\mathbf{x}, t), \phi_{j}\left(\mathbf{x}^{\prime}, t\right)\right]=\left[\pi_{j}(\mathbf{x}, t), \pi_{j}\left(\mathbf{x}^{\prime}, t\right)\right]=0 .}
\end{aligned}
$$

Suppose that Euclidean transformations $(\mathbf{a}, \mathbf{R})$ (where a stands for a translation and $\mathbf{R}$ for a rotation) of the field are induced by unitary transformations $U_{j}(\mathbf{a}, \mathbf{R})$ :

$$
\begin{aligned}
& U_{j}(\mathbf{a}, \mathbf{R}) \phi_{j}(\mathbf{x}, t) U_{j}^{-1}(\mathbf{a}, \mathbf{R})=\phi_{j}(\mathbf{R x}+\mathbf{a}, t) \\
& U_{j}(\mathbf{a}, \mathbf{R}) \pi_{j}(\mathbf{x}, t) U_{j}^{-1}(\mathbf{a}, \mathbf{R})=\pi_{j}(\mathbf{R} \mathbf{x}+\mathbf{a}, t)
\end{aligned}
$$

And suppose that the fields are related at some time $t$ by a unitary transformation $V(t)$ :

$$
\phi_{2}(\mathbf{x}, t)=V(t) \phi_{1}(\mathbf{x}, t) V^{-1}(t), \quad \pi_{2}(\mathbf{x}, t)=V(t) \pi_{1}(\mathbf{x}, t) V^{-1}(t)
$$

Then

$$
U_{2}(\mathbf{a}, \mathbf{R})=V(t) U_{1}(\mathbf{a}, \mathbf{R}) V^{-1}(t)
$$

Further, if there are unique normalizable states $\left|0_{j}\right\rangle$ invariant under Euclidean transformations

$$
U_{j}(\mathbf{a}, \mathbf{R})\left|0_{j}\right\rangle=\left|0_{j}\right\rangle
$$


then

$$
c\left|0_{2}\right\rangle=V(t)\left|0_{1}\right\rangle
$$

where $|c|=1$.

HHW theorem, Part II. Consider fields satisfying the hypotheses of Part I. Suppose that Poincaré transformations $(a, \Lambda)$ (where $\Lambda$ stands for a proper Lorentz transformation) of the fields are induced by unitary transformations $T_{j}(a, \Lambda)$ :

$$
T_{j}(a, \Lambda) \phi_{j}(x)=\phi_{j}(\Lambda x+a), \quad j=1,2
$$

Suppose further that the states $\left|0_{j}\right\rangle$ are Poincaré invariant

$$
T_{j}(a, \Lambda)\left|0_{j}\right\rangle=\left|0_{j}\right\rangle
$$

and that no states of negative energy exist. Then the first four vacuum expectation values of the two fields (the four-point "Wightman functions") are equal:

$$
\left\langle 0_{2}\left|\phi_{2}\left(x_{1}\right) \ldots \phi_{2}\left(x_{4}\right)\right| 0_{2}\right\rangle=\left\langle 0_{1}\left|\phi_{1}\left(x_{1}\right) \ldots \phi_{1}\left(x_{4}\right)\right| 0_{1}\right\rangle
$$

In the general case in which $\phi_{1}$ and $\phi_{2}$ are arbitrary fields, it can only be proven that the first four Wightman functions are equal. However, in the special case in which one of the fields-say, $\phi_{1}$-is a free field, it can be proven that all Wightman functions are equal, and hence that $\phi_{2}$ is also a free field. There are two routes to this result. One approach is to apply the Jost-Schroer theorem: Let $\phi$ be a Hermitian scalar field with the vacuum as a cyclic vector, and suppose that its two point vacuum expectation values coincide with those of a free field of mass $m>0$; then $\phi$ is a free field of mass $m$ (see Jost 1961). This result was extended to the zero-mass case by Pohlmeyer (1969). Alternatively, one could take the route of Greenberg (1959), who strengthened Part II of the HHW theorem by proving, under the same hypotheses, that if $\phi_{1}$ is a free field, then all of the $n$-point Wightman functions of the two fields are equal. The Wightman reconstruction theorem (Streater and Wightman 1964, p. 118) entails that, granting certain plausible assumptions, the two fields are unitarily equivalent; thus $\phi_{2}$ is also a free field. Either way, if $\phi_{1}$ is a free field, then $\phi_{2}$ is also a free field.

Like the heuristic version of Haag's theorem, the formal HHW theorem takes the form of a reductio argument. The conclusion that both fields are free fields is unacceptable because the intention is to represent an interacting field; therefore, at least one of the assumptions of the theorem must be dropped. In the HHW theorem, an obvious candidate is the assumption that at some $t$ 
there exists a unitary transformation $V(t)$ relating the fields. The analogous response to the heuristic version of Haag's theorem is to reject either the assumption that the vacuum state of the interacting field lies in $\mathcal{H}_{F}$ or the assumption that the full interaction Hamiltonian $H$ is a well-defined operator on $\mathcal{H}_{F}$ (or both). This explains why Haag's theorem is sometimes parsed as the statement that the physical vacuum, the Hamiltonian for the interacting field, and the Schrödinger dynamics for the interacting field do not exist in the Hilbert space of the free field $\mathcal{H}_{F}$ but require a different, "renormalized" Hilbert space $\mathcal{H}_{R} \cdot{ }^{13}$

Such a gloss, however, may be puzzling to the mathematically minded reader who will immediately point out that all infinite dimensional separable Hilbert spaces are the same (i.e. isomorphic), and that an isomorphism from the renormalized Hilbert space $\mathcal{H}_{R}$ of the interacting field-where $\left|0_{I}\right\rangle$ and the interacting field Hamiltonian $H$ naturally live-to $\mathcal{H}_{F}$ can be used to identify $\left|0_{I}\right\rangle$ and $H$ with corresponding objects in $\mathcal{H}_{F}$. This is correct; however, no such identification can make the respective representations of the CCR by the free and interacting fields unitarily equivalent.

Here is another way to appreciate this result, on the assumption that the vacuum states for the free and the interacting fields are both no-particle states. Suppose for sake of reductio that the annihilation operators $a_{F}$ and $a_{I}$ for the free and interacting fields respectively are connected by a transformation of the form $a_{I}(f)=U a_{F}(f) U^{-1}$ for a unitary $U \cdot{ }^{14}$ Then the vacuum state for the interacting field could be defined as $\left|0_{I}\right\rangle:=U\left|0_{F}\right\rangle$ since $a_{I}(f)\left|0_{I}\right\rangle=U a_{F}(f) U^{-1} U\left|0_{F}\right\rangle=0$. So the statement that $\left|0_{I}\right\rangle \notin \mathcal{H}_{F}$ should be construed as a short hand way of saying that the Hilbert space descriptions of the free and interacting fields belong to unitarily inequivalent representations of the CCR.

The mathematically minded reader may still be troubled by a second puzzle about how a Hilbert space description $\mathcal{H}_{R}$ of the interacting field is possible. For if $H_{F}+H_{I}$ is a well-defined operator on $\mathcal{H}_{R}$ then so it would seem is $H_{F}$, and so, contrary to what Haag's theorem was supposed to show, the free and the physical vacuum, as well as the free and interacting field dynamics, can be described within the same Hilbert space. The fallacy that generates this puzzle is the assumption that the ' + ' in $H_{F}+H_{I}$ should be taken to mean that each operator in the formal sum is separately well-defined on $\mathcal{H}_{R}$, whereas in fact only the combined operator has meaning. Indeed, this moral has also been taken as a statement of Haag's theorem (see Klauder 2000, p. 131). 


\section{Taking stock}

Before proceeding to a discussion of the implications of Haag's theorem, a number of comments are in order. They fall into two groups of three. The first group concerns the proof of the HHW theorem and the second group concerns the limitations of all versions of Haag's theorem.

To begin with the first group, the first thing to note about the HHW version of Haag's theorem recapitulated in the preceding section is that it relies heavily on the Wightman formalism for QFT. In particular, the hypotheses of the theorem are used to show that the Wightman functions are boundary values of analytic functions and that the values of the first four Wightman functions are uniquely determined from their values at equal times. However, Haag's theorem is emphatically not a hostage to the Wightman formalism, as is shown by the fact that results to the same effect can be proven by alternative means. Another style of proof using the Yang-Feldman representation of quantum field is given by Lopuzanski $(1961,1965)$. Yet another proof based on the algebraic approach to QFT is given by Emch (1972, pp. 247-53). It is safe to conclude that Haag's theorem is a robust result that is not dependent on any particular formalism for QFT.

Second, while Poincaré covariance of the fields and the Poincaré invariance of the vacuum state are important ingredients of the HHW theorem, Wightman himself played down the importance of relativistic invariance:

The strange representations ${ }^{15}$ associated with Haag's theorem are, in fact, an entirely elementary phenomenon and appear as soon as a theory is euclidean invariant and has a Hamiltonian which does not have the no-particle state as a proper vector [vacuum polarization]. This will happen whether or not the theory is relativistically invariant and whether or not there are ultraviolet problems in the theory. (1967, p. 255)

Wightman is correct insofar as vacuum polarization may occur in any two theories with an infinite number of degrees of freedom, relativistic or not; consequently, unitarily inequivalent representations must be used for these theories. However, the HHW theorem licenses the stronger statement that in a relativistic theory satisfying its assumptions, vacuum polarization must occur. Relativistic invariance need not be invoked to prove Part I of the HHW theorem; Euclidean invariance is sufficient. This result implies that, 
if the free and interacting representations are unitarily equivalent, then the vacuum for the free field $\left|0_{F}\right\rangle$ is also (up to phase) the vacuum for the interacting field; that is, vacuum polarization does not occur, $\left(H_{F}+H_{I}\right)\left|0_{F}\right\rangle=0$. However, Poincaré invariance is used in the proof of Part II of the HHW theorem. In informal terms, Part II establishes that in a relativistic QFT, $\left(H_{F}+H_{I}\right)\left|0_{F}\right\rangle=0$ (the nonoccurrence of vacuum polarization) implies that $\left(H_{F}+H_{I}\right)$ must be a free Hamiltonian. The relativistic assumptions in Part II of the HHW theorem are needed to derive the equality of the first four vacuum expectation values for arbitrary times from the equality of the vacuum expectation values for equal times. To do this, Hall and Wightman rely on the fact that for a Poincaré covariant field and Poincaré invariant vacuum, the vacuum expectation values are the boundary values of analytic functions (Hall and Wightman 1957). Intuitively, the properties of arbitrary time vacuum expectation values do not follow from equal-time vacuum expectation values in a Galilean invariant QFT because time is absolute. In a relativistic QFT, Poincaré invariance constrains arbitrary time vacuum expectation values because a time translation is equivalent to a Lorentz boost plus a space translation plus a Lorentz boost. In sum, relativistic invariance is an essential ingredient of the HHW version of Haag's theorem.

Furthermore, it is known that Euclidean invariance plus the existence of an infinite number of degrees of freedom is not sufficient to force the use of inequivalent representations. This is demonstrated by the existence of counterexamples: there exist Galilean invariant QFT's in which vacuum polarization does not occur and in which non-trivial interactions may be represented on the Fock representation for a free field (see Lévy-Leblond 1967, 1971; Hepp 1969, Ch. 3). ${ }^{16}$ However, it is not established that relativistic invariance is essential in forcing the use of inequivalent representations, and as far as we are aware, it is open that there are examples of Galilean QFTs that do display vacuum polarization.

Third, the role of an infinite number of degrees of freedom was not made explicit in the above sketch of the HHW theorem. Of course, one knows from the Stone-von Neumann theorem that when the system at issue has only a finite number of degrees of freedom the HHW will not apply. But which assumptions of the theorem fail? For systems with a finite number of degrees of freedom, there can exist multiple normalizable states invariant under Euclidean translations (Hall and Wightman 1957, p. 125; Streit 1969, pp. 679-670). Thus, the assumption of Part I of the HHW theorem that $\left|0_{j}\right\rangle$ are the unique normalizable states invariant under Euclidean transformations 
is physically unreasonable for such systems.

The second group of comments concerns the limitations, not just of the HHW theorem, but also of other versions of Haag's theorem. The first and most obvious comment is that the crucial assumption of spatial translation invariance can be killed in various ways, e.g. by "putting the system in a box" and using periodic boundary conditions or by having the system interact with an appropriate external potential (see Reed and Simon 1979, Sec. XI.15). In such cases Haag's theorem does not apply and the conclusion of the theorem can be escaped.

Second, Haag's theorem relies on the ETCCR. The mathematically more precise expression of the ETCCR in (4) assumes that spatial-smearing (i.e. smearing $\phi$ and $\pi$ with suitable test functions $f$ of compact support on a spacelike hyperplane of Minkowski spacetime) is sufficient to produce welldefined operators $\phi(f, t):=\int \phi(x, t) f(x) d x$ and $\pi(f, t):=\int \pi(x, t) f(x) d x$. In reaction to Haag's theorem there were rumblings that perhaps smearing with functions of time as well as space is required and, thus, that equal-time CCR are not a suitable basis for treating interacting fields (Streater and Wightman 1964, p. 168; Bogoliubov et. al. 1975, p. 561). These suspicions were substantiated to some extent for boson fields by the work of Baumann (1987, 1988). Suppose that the sharp-time neutral scalar field $\phi(f, t)$ and is conjugate momentum $\pi(f, t)$ exist for all times and give an irreducible representation of the ETCCR. Then using plausible technical assumptions, Baumann showed that in a spacetime of dimension $(n+1)$ with $n>3$ the field is a free field. The same is true for $n=3$ with the possible exception of the $\left(\phi^{4}\right)_{3+1}$ field ${ }^{17}$ And for $n=2$ the ETCCR are fulfilled by the $\left(\phi^{4}\right)_{2+1}$ field but no other save possibly for $\left(\phi^{6}\right)_{2+1}$. Powers (1967) proved even more restrictive result for Fermion fields. Suppose that a sharp-time Fermion field and its conjugate momentum field exist for all times, give an irreducible representation of the equal-time canonical anti-commutation relations (ETCAR), and are covariant with respect to the Poincaré group. Suppose also that there is a unique Poincaré invariant vacuum state and that this state is cyclic with respect to the sharp-time fields. Then using plausible technical assumptions, Powers showed that the field is a free field.

Although Baumann's results limit the applicability of Haag's theorem, they do not undercut the main moral, viz. in the program of trying to construct mathematically well-defined Hilbert space representations for relativistic quantum fields, unitarily equivalent representations will have to come into play. ${ }^{18}$ (This moral extends beyond interacting fields since, as will be 
discussed below, free fields of different masses possess unitarily inequivalent Fock representations.) Subsequent no-go results do not show that field theorists do not have to worry about Haag's theorem because some of its assumptions do not hold in all cases of interest; rather, what the subsequent results show that even more assumptions have to be abandoned in order to obtain well-defined Hilbert space descriptions of interacting fields.

Third, Haag's theorem only captures one of the reasons why unitarily inequivalent representations of the CCR come into play in the description of interacting fields. In the physicists' lingo, the move from one inequivalent representation to another is marked by divergences. Haag's theorem is concerned with infrared divergences that are associated with Euclidean invariance and the infinite volume of space. These divergences can be tamed, and Haag's theorem rendered inapplicable, by compactifying space (e.g. by identifying $\mathbf{x}$ with $\mathbf{x}^{\prime}$ when $\mathbf{x}^{\prime}=\mathbf{x} \bmod$ some fixed vector). However, such a theory may still be plagued by divergences of other types; for example, irreducible representations for a free scalar field and a $\phi^{4}$ field in spatially compactified $(2+1)$-dim Minkowski spacetime are known to belong to inequivalent representations because of ultraviolet divergences that occur at high energy-momentum (Jaffe 1999, note 2, p. 140).

To summarize, while it is well to recognize all of the caveats and nuances listed above, they should not cause one to lose sight of the key point of relevance here; namely, Haag's theorem was instrumental in convincing physicists that inequivalent representations of the CCR are not mere mathematical playthings but are essential in the description of quantum fields-in particular, because the choice of an appropriate representation depends on the dynamics of the field. We will have more to say about the choice problem in Section 7. But first we take up the implications of Haag's theorem for the interaction picture and for scattering theory.

\section{The interaction picture}

In the canonical approach to QFT, the interaction picture is an important means of representing interactions and extracting predictions that can be compared with experimental results. The conundrum posed by Haag's theorem for the interaction picture was pithily stated by Roman (1969): "The most sobering consequence of Haag's theorem is that the interaction picture of canonical field theory cannot exist unless there are no interactions" (p. 
391). To see how this comes about we briefly recapitulate the assumptions underlying the interaction picture in QFT.

Split the full interaction Hamiltonian $H$ into free and interacting parts, $H=H_{F}+H_{I}$. In analogy to Dirac's interaction picture formalism for ordinary QM, the interaction picture in canonical QFT uses an irreducible representation of the ETCCR in which the evolution of the states is governed by $H_{I}$ and the evolution of the operators is governed by $H_{F}$. This picture is intermediate between the Schrödinger picture-in which the states evolve under the full Hamiltonian $H$ and the operators are stationary-and the Heisenberg picture-in which the states are stationary and the operators evolve under the full Hamiltonian $H$. In particular, in the interaction picture the evolution of the field operators is generated by $H_{F}$, so the fields are free.

By stipulation, the interaction picture (indicated by the subscript ${ }_{I}$ ) coincides with the Heisenberg picture (indicated by the subscript ${ }_{H}$ ) at time $t_{0}$ :

$$
\begin{gathered}
\phi_{I}\left(\mathbf{x}, t_{0}\right)=\phi_{H}\left(\mathbf{x}, t_{0}\right) ; \pi_{I}\left(\mathbf{x}, t_{0}\right)=\pi_{H}\left(\mathbf{x}, t_{0}\right) \\
\left|\psi\left(t_{0}\right)\right\rangle_{I}=|\psi\rangle_{H}
\end{gathered}
$$

Let $V\left(t_{1}, t_{2}\right)$ represent the unitary evolution operator from $t_{1}$ to $t_{2}$ for the interaction picture states: $\left|\psi\left(t_{2}\right)\right\rangle_{I}=V\left(t_{2}, t_{1}\right)\left|\psi\left(t_{1}\right)\right\rangle_{I}$. Since in the interaction picture the evolution of the states is generated by $H_{I}$,

$$
\begin{aligned}
V\left(t_{1}, t_{2}\right) & =e^{-i H_{I}\left(t_{2}-t_{1}\right)} \\
& =e^{i H_{F}\left(t_{2}-t_{1}\right)} e^{-i H\left(t_{2}-t_{1}\right)}
\end{aligned}
$$

It follows from (14) and the dynamics of the states and operators in the two pictures that, at any time $t$, the representations are related by $V\left(t, t_{0}\right)$ :

$$
\begin{gathered}
\phi_{I}(\mathbf{x}, t)=V^{-1}\left(t, t_{0}\right) \phi_{H}(\mathbf{x}, t) V\left(t, t_{0}\right) \\
\pi_{I}(\mathbf{x}, t)=V^{-1}\left(t, t_{0}\right) \pi_{H}(\mathbf{x}, t) V\left(t, t_{0}\right) \\
|\psi(t)\rangle_{I}=V^{-1}\left(t, t_{0}\right)|\psi\rangle_{H}
\end{gathered}
$$

The quantity that is of physical interest is the amplitude

$$
\begin{aligned}
{ }_{H}\left\langle\chi_{\text {in }} \mid \psi_{\text {out }}\right\rangle_{H} & =\lim _{t_{1} \rightarrow-\infty, t_{2} \rightarrow+\infty}\left[{ }_{I}\left\langle\chi\left(t_{1}\right)\left|V\left(t_{1}, t_{0}\right) V^{-1}\left(t_{2}, t_{0}\right)\right| \psi\left(t_{2}\right)\right\rangle_{I}\right] \\
& :={ }_{I}\left\langle\chi_{\text {in }}|S| \psi_{\text {out }}\right\rangle_{I}
\end{aligned}
$$


The limits $t \rightarrow \pm \infty$ are taken for convenience: at these times particles are assumed to be infinitely far apart and therefore not interacting; thus, the states $|\chi(-\infty)\rangle_{I},|\psi(+\infty)\rangle_{I}$ are free states (Schweber 1962, p. 318). In the interaction picture, this means that at $t= \pm \infty$ the representation is the Fock representation for the free field $\phi_{I}(\mathbf{x}, t) . S$-matrix elements in the interaction picture can be evaluated using perturbative methods. An account of how perturbation theory is applied is the beyond the scope of this paper, ${ }^{19}$ but it should be noted that the application makes essential use of the interaction picture. The terms of the perturbation series are evaluable by virtue of the fact that at $t= \pm \infty$ the action of $\phi_{I}$ on the vacuum can be calculated. In contrast, even at $t= \pm \infty$, we do not have a concrete Hilbert space representation for $\phi_{H}$, so we do not know how to evaluate vacuum expectation values of $\phi_{H}$.

All of the assumptions of the HHW are adopted in this canonical approach to treating interactions. Some of the assumptions of the theorem are general presuppositions of canonical QFT while others are specific to the interaction picture. The assumption that the fields give irreducible representations of the ETCCR's, is a standard assumption of this approach (see Schweber (1962, pp. 650-651)). ${ }^{20}$ Likewise, it is typically assumed that Poincaré (and hence Euclidean) transformations are induced by unitary operators (see Schweber (1962, p. 265)). The assumption that there are no negative energies is justified on physical grounds. That the fields transform appropriately under Euclidean and Poincaré transformations is taken to be a requirement of a QFT with or without interactions (see Schweber (1962, p. 265)). The assumption that there exist unique normalizable vacuum states that are invariant under Poincaré transformations follows from Wigner's classification of representations of the inhomogeneous Lorentz group (Schweber 1962, pp. 165-166; Streater and Wightman 1964, pp. 21-22, 27-29). This assumption holds at all times for the Heisenberg picture; for the interaction picture, it certainly holds at $t= \pm \infty$, when the representation is taken to be a Fock representation so the no-particle state is the vacuum state for the field $\phi_{I}$, which is governed by $H_{F}$. The assumption that there is some time at which the fields in the representations are related by a unitary transformation, holds at all times in virtue of the fact that at each time $t$ the fields in the representations are related by the unitary transformation $V\left(t, t_{0}\right)$. Thus, at times $t= \pm \infty$, all the assumptions of the theorem hold for the Heisenberg picture representation, which represents an interaction, and for the interaction picture representation, which is a Fock representation for a free system. 
The conundrum is now evident: the interaction picture presupposes all of the assumptions needed for the HHW theorem; but this theorem shows that the interaction picture cannot be used to represent a non-trivial interaction. And yet the interaction picture and perturbation theory work. Some explanation of why they work is called for. We suspect that the full explanation will have a number of different pieces. Here we will only point to what we believe is a critical piece in the overall scheme.

The HHW theorem demonstrates that the interaction picture is predicated on an inconsistent set of assumptions. In response to this reductio of the assumptions, at least one must be abandoned. Note, however, that if the interaction picture is to fulfill its purpose of enabling the evaluation of the scattering matrix elements via perturbation theory, some key assumptions must be retained. In particular, the unitary equivalence of the Heisenberg and interaction pictures is essential. Note also that the problem brought to light by Haag's theorem is not directly related to the employment of perturbation theory as an approximation method; all of the assumptions of the HHW theorem are embraced before the perturbation series is even introduced.

To see how the effectiveness of the interaction picture and perturbation theory can be explained in the face of Haag's theorem, consider the simple case of two free neutral scalar fields $\phi_{m_{1}}$ and $\phi_{m_{2}}$, with masses $m_{1} \neq m_{2}$, and their conjugate momentum fields $\pi_{m_{1}}$ and $\pi_{m_{2}}$. If $m_{2}=m_{1}+\delta m$, one might seek to treat the $\phi_{m_{2}}$ field in the interaction picture by writing its Hamiltonian as the sum of the Hamiltonian for $\phi_{m_{1}}$ plus a term of the form $\int \delta m: \phi_{m_{1}}^{2}(\mathbf{x}): d^{3} x$. Then $\phi_{m_{1}}$ plays the role of $\phi_{I}$ and $\phi_{m_{2}}$ plays the role of $\phi_{H}$. But irreducible representations of these fields are unitarily inequivalent, as shown by Haag type theorems. The heuristic proof derives from the fact that the (formal) Hamiltonian for the $\phi_{m_{2}}$ field polarizes the vacuum of the $\phi_{m_{1}}$ field. A more rigorous proof can be found in Reed and Simon (1975, Theorem X.46). So the situation is much worse than the above quote from Roman indicates: the interaction picture cannot exist even if there are no interactions!

Nevertheless, there is a perfectly good sense in which the interaction picture and perturbation theory do work for free fields of different masses, at least for physical quantities that matter for explaining experimental outcomes. This is because although Haag's theorem undercuts global unitary equivalence, it is compatible with local unitary equivalence. We quote the version of local equivalence given in Reed and Simon (1975) 
Given any bounded region $B \subset \mathbb{R}^{3}$ and the free fields $\phi_{m_{1}}, \pi_{m_{1}}$ and $\phi_{m_{2}}, \pi_{m_{2}}$ acting on the respective Hilbert spaces $\mathcal{H}_{1}$ and $\mathcal{H}_{2}$, there is unitary map $V_{B}: \mathcal{H}_{1} \rightarrow \mathcal{H}_{2}$ such that $V_{B} \phi_{m_{1}}(f) V_{B}^{-1}=$ $\phi_{m_{2}}(f)$ and $V_{B} \pi_{m_{1}}(f) V_{B}^{-1}=\pi_{m_{2}}(f)$ for all suitable test functions $f$ with support in $B$. (p. 329)

Thus, insofar as one is concerned with explaining the results of experiments on local observables for free fields of different masses, Haag's theorem poses no obstacle to using perturbation theory. Of course, these remarks do nothing to address questions about whether the local perturbation expansion is defined term by term, whether the expansion converges etc. But these issues have nothing directly to do with the bite of Haag's theorem since they arise any time a perturbation series is employed and, as noted above, the use of a perturbation series as an approximation method is not the source of the problem brought to light by Haag's theorem.

What is true of the case of free fields of different masses is also true of a free field with mass $m$ and Hamiltonian $H_{F}$ and the renormalized $\left(\phi^{4}\right)_{1+1}$ field with formal Hamiltonian $H=H_{F}+\lambda \int: \phi(x)^{4}: d x$ (see Glimm and Jaffe 1968, 1970a, 1970b). ${ }^{21}$ This result was to be expected: the normal-ordered $\phi^{4}$ field in $(1+1)$-dim Minkowski spacetime does not have ultraviolet divergences, and thus it is not surprising that local unitary equivalence to the free field is obtained since restricting to bounded regions of space quashes infrared divergences. Unfortunately, in higher spacetime dimensions the ultraviolet divergences are more severe and local unitary equivalence does not hold (Jaffe 1999, note 2, p. 140). However, Haag's theorem is not responsible for the problems created by ultraviolet divergences, so solving them is beyond the scope of this paper. ${ }^{22}$

If the interaction picture were valid, it could be used as a basis for scattering theory; in particular, if $(17 \mathrm{a}, \mathrm{b})$ were valid, the transformation $V\left(t, t_{0}\right)$ could be used to construct to construct the $S$-matrix:

$$
S(-\infty,+\infty)=\lim _{t_{1} \rightarrow-\infty, t_{2} \rightarrow+\infty} V\left(t_{1}, t_{0}\right) V^{-1}\left(t_{2}, t_{0}\right)
$$

Unfortunately, the HHW theorem blocks this route to scattering theory for quantum fields. But fortunately, there is another approach that does not rely on the interaction picture. Before turning to this matter we want to comment on the hyperventilation of philosophers over Haag's theorem (recall the examples cited in the Introduction). The fact that Haag's theorem undermines 
a standard textbook treatment of interactions (i.e., the interaction picture) is one of the main causes of this hyperventilation. Indeed, the remarks by philosophers to the effect that Haag's theorem seems to show that there is no consistent formalism for interacting quantum field theory are charitably interpreted as referring to this fact. One way to soothe the hyperventilation is to take note of the existence of the strategy outlined above for explaining why, in spite of Haag's theorem, the interaction picture works. Another way is to note that various problems in QFT can be treated without the use of the interaction picture. One example is the existence of alternative approaches to scattering theory (such as the one described in the next section). Another example is the (limited) success of constructive field theory in producing representations in which non-perturbative calculations can be carried out.

\section{Scattering theory}

To describe how scattering theory in QFT can be formulated without running afoul of Haag's theorem, it will be helpful to begin with a brief review of scattering theory in ordinary QM. Suppose that the free and interacting dynamics are given respectively by $U_{F}(t):=e^{-i H_{F} t}$ and $U(t):=e^{-i H t}, H=$ $H_{F}+H_{I}$. One would like to be able to say that, in some appropriate sense, as $t \rightarrow \pm \infty$ the free and interacting dynamics coincide. The strongest sense of convergence is that $\left(U(t)-U_{F}(t)\right)$ goes to zero in the operator norm topology as $t \rightarrow \pm \infty$. But this would mean that $U^{*}(t) U_{F}(t) \rightarrow 1$ in the operator norm topology, which can happen if and only if $H=H_{F}$ (Reed and Simon 1979, p. 386).

To achieve a positive result it is necessary to use a weaker sense of convergence and, perhaps, also to require convergence only for a limited subset of states ("scattering states"). To this end, associate with the Hamiltonian $H$ governing interactions a set of scattering states $M_{S}(H) \subset \mathcal{H}$ and with the free Hamiltonian $H_{F}$ two sets of scattering states $M_{S}^{ \pm}\left(H_{F}\right) \subset \mathcal{H}$. What we would like to obtain is the result that for every $|\psi\rangle \in M_{S}(H)$ there are $|\psi\rangle_{+} \in M_{S}^{+}\left(H_{F}\right)$ and $|\psi\rangle_{-} \in M_{S}^{-}\left(H_{F}\right)$ such that the following limits exist:

$$
\lim _{t \rightarrow \pm \infty} \| U_{F}(t)|\psi\rangle_{ \pm}-U(t)|\psi\rangle \|=0
$$

This condition holds just in case

$$
\lim _{t \rightarrow \pm \infty} \| U^{*}(t) U_{F}(t)|\psi\rangle_{ \pm}-|\psi\rangle \|=0
$$


The latter is just the condition that the wave operators $\Omega^{ \pm}$exist in the strong limit sense:

$$
\Omega^{ \pm}:=s-\lim _{t \rightarrow \mp \infty} U^{*}(t) U_{F}(t) E_{S}^{ \pm}\left(H_{F}\right)
$$

where $E_{S}^{ \pm}\left(H_{F}\right)$ projects onto $M_{S}^{ \pm}\left(H_{F}\right)$. And one would also like asymptotic completeness in the sense that each of $M_{S}^{ \pm}\left(H_{F}\right)$ spans $\mathcal{H}$. The existence of the wave operator and the satisfaction of asymptotic completeness in ordinary QM is proven for various cases, the simplest being that where $H_{F}=-\Delta$ and $H=H_{F}+V(r)$ with $V(r)$ vanishing faster than $1 / r^{2}$ as $r \rightarrow \infty$ (see Prugovečki 1981).

In relativistic QFT, Haag's theorem makes hash out of this picture since, in informal terms, the theorem indicates that $H$ and $H_{F}$ are not defined on the same Hilbert space. Moving from strong to weak convergence does not help since the latter, like the former, assumes that the Hamiltonians of the free and interacting fields are defined on the same Hilbert space. ${ }^{23}$ More formally, the HHW theorem undermines this approach in precisely the same manner that it undermines the interaction picture. This can be seen from the fact that the wave operators $\Omega^{ \pm}$can be expressed in terms of $V\left(t, t_{0}\right)$, the unitary evolution operator for the interaction picture introduced in the preceding section:

$$
\begin{aligned}
V(t, 0) & =e^{i H_{F} t} e^{-i H t} \\
\Omega^{ \pm} & =s-\lim _{t \rightarrow \mp \infty} V^{*}(t, 0)
\end{aligned}
$$

This conundrum is resolved by Haag-Ruelle scattering theory (see Haag 1958 and Ruelle 1962). Because the details of this approach are so complicated and require so many technical niceties, only a skeleton outline will be presented here. The basic move is to define in the Hilbert space $\mathcal{H}$ of the interacting field surrogates of the free field states in the asymptotic regimes $t \rightarrow-\infty$ and $t \rightarrow+\infty$. Denote the subspaces spanned by these surrogate states $\mathcal{H}_{\text {in }}$ and $\mathcal{H}_{\text {out }}$ respectively. Demonstrating the existence of these surrogate states permits the definition of an identification map $\mathcal{I}$ from a dense subset of the Hilbert space $\mathcal{H}_{F}$ of the free field to the surrogate asymptotic states in $\mathcal{H}$. The goal is then to show that analogues $\tilde{\Omega}^{ \pm}$of the wave operators of (23) exist in the strong limit sense:

$$
\tilde{\Omega}^{ \pm}:=s-\lim _{t \rightarrow \mp \infty} U^{*}(t) \mathcal{I} U_{F}(t)
$$


and that $\tilde{\Omega}^{+}$and $\tilde{\Omega}^{-}$define partial isometries from $\mathcal{H}_{F}$ to $\mathcal{H}_{\text {in }}$ and $\mathcal{H}_{\text {out }}$ respectively. Surrogates $\varphi_{\text {in }}$ and $\varphi_{\text {out }}$ of the asymptotic free fields can be defined on $\mathcal{H}_{\text {in }}$ and $\mathcal{H}_{\text {out }}$ respectively in terms of the operators creating and destroying the asymptotic particle states. If asymptotic completeness holdsi.e., $\mathcal{H}_{\text {in }}=\mathcal{H}_{\text {out }}=\mathcal{H}$-then the $i n$ and out fields are connected by an $S$-matrix, $\varphi_{\text {out }}=S^{-1} \varphi_{\text {in }} S$, where $S(-\infty,+\infty):=\tilde{\Omega}^{+}\left(\tilde{\Omega}^{-}\right)^{*}$. This formalism is not subject to Haag's theorem because-unlike the interaction picture-it neither posits nor entails the existence of a unitary transformation connecting $\mathcal{H}$ (or $\mathcal{H}_{\text {in }}$ or $\mathcal{H}_{\text {out }}$ ) to $\mathcal{H}_{F}$ that relates the interacting field to a free field.

In sum, although Haag's theorem poses an obstacle to transferring the usual way of doing scattering theory in ordinary QM to relativistic QFT, the Haag-Ruelle approach shows how to maneuver around this obstacle and to obtain in QFT analogues for most of the significant features of ordinary scattering theory. ${ }^{24}$

To our knowledge, the developers of Haag-Ruelle scattering theory nowhere present it as a response to Haag's theorem. But even if this was not the intent of the developers, our claim is that for understanding the foundations of QFT it is fruitful to see the Haag-Ruelle approach as bridging a conundrum created by Haag's theorem. In support of this interpretation we can cite the best of authorities. In the preface to their exposition of Haag-Ruelle scattering theory, Reed and Simon (1979) comment that

The first conceptual problem that must be faced is that there is no natural "free" dynamics for the interacting dynamics to approach as $t \rightarrow \pm \infty$. This is somewhat surprising since one might think that the Hamiltonian $\left[H_{F}\right]$ of this free field would play the role of of free dynamics. In the first place, the two Hamiltonians $\left[H_{F}\right.$ and $\left.H_{I}\right]$ act on two different Hilbert spaces ... a general result known as Haag's theorem. (p. 317) ${ }^{25}$

\section{Fock and non-Fock representations}

One of the better summaries of Haag's theorem to be found in the philosophical literature is in Heathcote (1989, pp. 91-92). But we want to take issue with the claim made there that Haag's theorem shows the necessity of using non-Fock representations. It is not hard to see how this claim might have been inferred from discussions of Haag's theorem in the physics literature. ${ }^{26}$ But the claim is suspect. Haag's theorem does indubitably show 
that QFT cannot be done in one "universal receptacle," i.e. a single unitary equivalence class of representations. A fortiori QFT cannot be done within a fixed Fock space, in particular, the Fock space for, say, a free neutral scalar field of mass $m$. But does Haag's theorem itself show that interacting fields require non-Fock representations? As noted above, Haag's theorem shows that an irreducible representation of the CCR for a neutral scalar field of mass $m^{\prime} \neq m$ is unitarily inequivalent to that for the field of mass $m$ although, of course, both representations are Fock representations. Similarly, a Haag style argument applies to the $\phi^{2}$ field of mass $m$ since the (formal) Hamiltonian for this field "polarizes the vacuum" of the free field of mass $m$. But in $(n+1)$-dim Minkowski spacetime with $1 \leq n \leq 3$, the standard renormalization procedure applied to the $\phi^{2}$ field leads to a representation that is unitarily equivalent to the Fock representation of the free field with mass $m^{\prime}=\sqrt{m^{2}+\lambda}$, where $\lambda$ is the self-coupling constant of the $\phi^{2}$ field (see Ginibre and Velo 1970). By the previously mentioned result for free fields of different masses, this Fock representation of the renormalized $\phi^{2}$ field of mass $m$ is unitarily inequivalent to Fock representation of the $m$ field. Indeed, the renormalization procedure should yield this result since, classically, the $\phi^{2}$ field obeys $\square \phi-m^{2} \phi=\lambda \phi$, which is equivalent to the Klein-Gordon equation of a free scalar field with renormalized mass $m^{\prime}=\sqrt{m^{2}+\lambda}$. In sum, Haag's theorem applies to cases where the inequivalent representations are both Fock representations. Thus, if it is true that non-trivial interactions have to be described using non-Fock representations, considerations beyond those invoked in Haag's theorem have to be invoked. Before we turn to such considerations, we need to say in more detail just what is involved in a Fock representation.

As detailed in the Appendix, a Fock representation of the CCR is characterized by the existence of a vacuum state which is annihilated by all of the annihilation operators $a(f)$ and from which a dense set of states can be built by acting on the vacuum with creation operators $a^{*}(f)$, where $f$ ranges over elements of the complexified test function space (or "one particle Hilbert space"-see Appendix). Such a representation contains a self-adjoint operator $N$, defined on some dense domain, having the spectrum $\{0,1,2, \ldots\}$ and satisfying the commutation relation $\left[N, a^{*}(f)\right]=1$. This operator is referred to as the total number operator. The existence of an operator $N$ having these properties guarantees that the representation is unitarily equivalent up to multiplicity to a Fock representation (Dell'Antonio and Doplicher 1967). For a free field, this $N$ counts the number of quanta of excitation of the field. The 
failure of a representation to admit a total number operator means, loosely speaking, that there are too many quanta of excitation to count. For this reason such representations were dubbed "myriotic" by Friedrichs (1953), who was the first to investigate them.

One source of confusion about whether Haag's theorem implies that nonFock representations have to be used derives from a slide between the absolute and relative senses of "myriotic representations." How easy it is to be caught in the slide is illustrated by a toy example from Bogoliubov et al. (1975). Consider the transformations of canonical variables $p_{k}, q_{k}$ given by

$$
p_{k}^{\prime}=\exp \left(\alpha_{k}\right) p_{k}, \quad q_{k}^{\prime}=\exp \left(-\alpha_{k}\right) q_{k}
$$

where the $\alpha_{k}$ are positive constants. The associated "annihilation" and "creation" operators are defined by

$$
a_{k}:=\frac{1}{\sqrt{2}}\left(p_{k}-i q_{k}\right), \quad a_{k}^{*}:=\frac{1}{\sqrt{2}}\left(p_{k}+i q_{k}\right),
$$

The effect of (26) is to transform these operators as follows:

$$
\begin{aligned}
a_{k}^{\prime} & =\cosh \left(\alpha_{k}\right) a_{k}+\sinh \left(\alpha_{k}\right) a_{k}^{*} \\
a_{k}^{\prime *} & =\cosh \left(\alpha_{k}\right) a^{*}+\sinh \left(\alpha_{k}\right) a_{k}
\end{aligned}
$$

From the fact that the $p_{k}$ and $q_{k}$ obey the standard CCR, it follows that

$$
\left[a_{m}, a_{n}\right]=0=\left[a_{m}^{*}, a_{n}^{*}\right], \quad\left[a_{m}, a_{n}^{*}\right]=\delta_{m n} I
$$

The primed creation and annihilation operators defined by (28) also obey (29) when they are substituted for their unprimed counterparts. Now in the case of ordinary QM, where the index $k$ takes on only a finite number of values, the transformations (28) are unitarily implementable. But in the case of an infinite number of degrees of freedom the $\alpha_{k}$ can be chosen so that the series $\sum_{k=1}^{\infty} \sinh ^{2}\left(\alpha_{k}\right)$ diverges, in which case the transformations (28) are not unitarily implementable. But-and this is the crucial point-both the primed and unprimed creation and annihilation operators can figure in Fock representations. In the case where the series $\sum_{k=1}^{\infty} \sinh ^{2}\left(\alpha_{k}\right)$ diverges, these Fock representations are relatively myriotic: e.g. if the vacuum state in the 
unprimed Fock representation is $|0\rangle$, then a formal calculation of the expectation value of the primed total particle number operator $N^{\prime}=\sum_{k=1}^{\infty} a_{k}^{\prime *} a_{k}^{\prime}$ gives $\left\langle 0\left|N^{\prime}\right| 0\right\rangle=\left\langle 0\left|\sum_{k=1}^{\infty} a_{k}^{\prime *} a_{k}^{\prime}\right| 0\right\rangle=\sum_{k=1}^{\infty} \sinh ^{2}\left(\alpha_{k}\right)=\infty$, which is to say, intuitively, that the unprimed vacuum contains an infinite number of primed quanta and, more precisely, that $N^{\prime}$ is not a well defined operator in the unprimed Fock space. ${ }^{27}$ Conversely, the unprimed total particle number operator $N$ is not a well-defined operator in the the primed Fock space.

Immediately following this example, Bogoliubov et al. (1975) say:

One might suppose that it is possible to use the [sic] Fock representation also in the theory of interacting fields ... But Haag's theorem shows this is not true: at any finite time we must use a strange representation of the CCR (in which, roughly speaking, each state contains an infinite number of particles). (p. 560)

As far as we have been able to determine, the term "strange representation" was first used in Haag (1955). In the quoted passage "strange" seems to be used to mean "myriotic" and the reader is being invited to slide between the absolute and relative senses of myriotic and conclude that Haag's theorem shows that an interacting field requires a representation of the CCR that is absolutely strange or myriotic, i.e. non-Fock.

Haag's theorem by itself does not entail that non-Fock representations must be used for interactions. But, regardless of what Haag's theorem does or does not show, one can ask whether is it true that interacting fields require non-Fock representations. By way of answering, start with a Hermitian scalar field $\phi$ and its conjugate momentum field $\pi$, and assume that they satisfy the CCR. Define the associated annihilation operators by $a(f):=[\phi(f)+$ $i \pi(f)] / \sqrt{2}$, where $f$ ranges over elements of the test function space. Require that there be a Fock vacuum state in the sense of a cyclic vector $|0\rangle$ such that $a(f)|0\rangle$ for all $f$. Then $|0\rangle$ must be a so-called quasi-free state (see Emch 1967, p. 242). (In algebraic QFT this can be made more precise. Start with a real linear symplectic space $(\mathcal{T}, \sigma)$ and build over this space the Weyl CCR algebra $\mathcal{W}(\mathcal{T}, \sigma)$, a $C^{*}$-algebra that encodes an exponentiated version of the CCR (see the Appendix). Corresponding to any positive symmetric bilinear map $\mu: \mathcal{T} \times \mathcal{T} \rightarrow \mathbb{R}$ satisfying a certain inequality (see Appendix), there is a state $\omega_{\mu}$ on $\mathcal{W}(\mathcal{T}, \sigma)$ determined by its action on the generators $W(f)$ of 
the algebra: $\omega_{\mu}(W(f))=\exp (-\mu(f, f) / 2)$. Such a state is called quasi-free. It is known that a pure quasi-free state determines a GNS representation that is a Fock representation with the GNS vector $\left|\Omega_{\mu}\right\rangle$ being the no-particle vacuum state (see Manceau and Verbeure 1968). The converse is also true: If the GNS representation determined by a state $\omega$ on $\mathcal{W}(\mathcal{T}, \sigma)$ is a Fock representation in which the GNS vector is the no-particle vacuum, then $\omega$ is a pure quasi-free state.)

The significance of this result lies in the fact that, because of the Gaussian nature of a quasi-free state, the $n$-point functions of such a state have very special properties: all odd $n$-point functions vanish and the even $n$-point functions are sums of products of two point functions; thus, all the truncated $n$-point functions (a.k.a. connected $n$-point functions) vanish except for $n=2$. A proof that a local quantum field theory whose truncated $n$-point functions vanish beyond some some finite order cannot lead to non-trivial scattering was given by Greenberg and Licht (1963). In this sense, Fock representations are generally inappropriate for interacting fields. This is not to say, however, that they are never appropriate for describing interacting fields. Indeed, in cases where Haag's theorem does not apply, not only can interacting fields be given a Fock representation, but the Fock representation for the interacting field can be unitarily equivalent to that of the free field. One case in point is the $\phi^{4}$ field with $m>0$ on the cylindrical spacetime that results from "rolling up" $(1+1)$-dim Minkowski spacetime along the space axis; there is no ultraviolet divergence for the $\left(\phi^{4}\right)_{1+1}$ case, and the compactification of space squelches the infrared divergence.

We have devoted so much attention to the discussion of Fock representations because of their importance for the interpretation of QFT; in particular, they are relevant to the issue of whether the particle notion is essential to an understanding of the theory. The availability of a Fock representation is not sufficient to underwrite a particle interpretation; the latter requires a demonstration that the quanta of excitation of the field can and do, under appropriate circumstances, display particle-like behavior. But the availability of a Fock representation does seem to be a necessary condition; for if there is no total particle number operator then identifying the quanta of excitation with particles leads to the embarrassment that it is not meaningful to ask how many particles are present. Thus, the existence of cases where Fock representations are not available is a blow to those who want to think that particles have some fundamental status in QFT and a support to those who think particles have secondary if not epiphenomenal status in QFT. ${ }^{28}$ 


\section{The choice problem}

As a consequence of the Stone-von Neumann theorem one can say that in ordinary QM (and, more generally, in the quantum mechanics of systems with a finite number of degrees of freedom) the kinematics, as given by the CCR algebra, determines the representation up to unitary equivalence. Since a unitary transformation between representations preserves self-adjointness, eigenvalue spectra, and every other property that might affect physical predictions, unitary equivalence of representations of the CCR is surely sufficient for physical equivalence. It follows that in ordinary QM the choice of representation is one of convenience-one is free to exercise one's esthetic inclinations in choosing among the representations picked out by the kinematics, secure in the knowledge that the choice does not affect anything that could possibly be checked by experiment.

In QFT the kinematics, as given by the CCR algebra, does not determine the representation up to unitary equivalence. How then is the practitioner of QFT to choose among the vast array of unitarily inequivalent representations? Haag's theorem shows that inequivalent representations are not mere mathematical playthings but (as Wightman and Schweber (1955) noted) "arise in the most elementary examples of field theory" and, thus, that the choice problem has to be taken seriously.

The pioneers of the algebraic approach to QFT initially responded by denying that there was a real problem to be solved. The underpinnings of this attitude was supposed to be provided by Fell's theorem (see Appendix), which shows that different representations-even unitarily inequivalent representations-of a Weyl CCR algebra cannot be distinguished by the determination of a finite number of expectation values if the determinations are made with finite accuracy. But unless a severely operational perspective is adopted, the indistinguishability of representations guaranteed by Fell's theorem does not guarantee that the representations are genuinely physically equivalent. ${ }^{29}$

A more promising approach to solving the choice problem takes a cue from Haag's theorem, which has sometimes been glossed as saying that the choice of representation depends on the dynamics. Could it be that an appropriate representation not only depends on but is determined (up to unitary equivalence) by the dynamics? One sometimes encounters sentiments to this effect. For example, Florig and Summers (2000) write that "Roughly speaking, the kinematical aspects determine the choice of CCR-algebra, whereas 
the dynamics fix the choice of the representation of the given CCR-algebra in which to make the relevant, perturbation-free computations" (p. 452). ${ }^{30}$

The latter part of the remark might seem viciously circular since "dynamics" in the Schrödinger sense involves a Hamiltonian operator, which presupposes a representation. A way out of this problem is provided by the algebraic approach. Start with a one parameter group $\mathfrak{a}(t)$ of automorphisms of the Weyl CCR algebra, which group is to be thought of as giving the "Heisenberg dynamics" of the field. (In the case of the Klein-Gordon field this group will be a group of symplectic transformations of the symplectic vector space of real valued solutions of the classical Klein-Gordon equation; see Arageorgis et al. 2002.) Then look for a Hilbert space representation of the Weyl CCR algebra in which the action of $\mathfrak{a}(t)$ is implemented by a strongly continuous unitary group $U(t)$. The self-adjoint generator $H$ of $U(t)$ is the Hamiltonian that implements the Schrödinger dynamics of the field. One would like the representation to be such that $H$ is a positive operator. And one would like the the representation to contain a vacuum vector $|\Omega\rangle$ in the sense that $U(t)|\Omega\rangle=0$ and that $|\Omega\rangle$ is a cyclic vector. Proving the existence and uniqueness (up to unitary equivalence) of such a representation would constitute a demonstration that the dynamics determines a vacuum representation. There are theorems of this type for linear fields (see Weinless 1969 and Kay 1978). We are unaware of corresponding existence and uniqueness results for interacting fields.

In addition, another discovery of relativistic QFT shows that in general the dynamics cannot determine the representation up to unitary equivalence. This is the phenomenon of spontaneous symmetry breaking where there is a symmetry of the dynamics that is not unitarily implementable; here the choice of representation depends on the way the symmetry is broken as well as on the dynamics. Cases of spontaneous symmetry breaking include linear fields, such as the zero mass Klein-Gordon field. Spontaneous symmetry breaking provides another example of why inequivalent representations have to be taken seriously in QFT. But since it is not directly relevant to Haag's theorem it will not be discussed further here. ${ }^{31}$

The need to refer to features of the state in choosing a representation also points to a general strategy for resolution of the choice problem. By the GNS theorem, any state $\omega$ on a $C^{*}$-algebra in general, and a CCR algebra in particular, determines a representation that is the unique (up to unitary equivalence) cyclic representation that respects the expectation values specified by $\omega .^{32}$ The choice of representation now reduces to the choice of state. 
The choice of state may be guided by the desire to respect the dynamics. For example, finding a state $\omega$ on the Weyl CCR algebra that is invariant under the automorphism group $\mathfrak{a}(t)$ giving the algebraic Heisenberg dynamics guarantees that the GNS representation determined by $\omega$ satisfies all of the conditions enumerated above, save possibly for the positivity of the Hamiltonian. But the choice of state and, thus, the choice of representation may be guided by other factors as well.

\section{Conclusion}

Haag's theorem can be viewed as a reductio argument showing that it is not possible to apply a set of seemingly natural mathematical assumptions to interacting quantum fields. The initial reactions to the theorem varied. Perhaps the most darkly pessimistic reaction was that given in Freeman Dyson's (1956) Mathematical Reviews summary of Haag (1955). Haag's theorem and the work of van Hove (1952) that inspired it were taken to

make even clearer than before the fact that the Hilbert space of ordinary quantum mechanics is too narrow a framework in which to give a consistent definition to the operators of quantum field theory. It is for this reason that attempts to build a rigorous basis for field theory within the Hilbert-space framework ... always stop short of any non-trivial examples. The question, what kind of enlarged framework would make consistent definitions possible, is the basic unsolved problem of the subject.

A less pessimistic reaction was championed by Wightman and his collaborators (and is endorsed by us): namely, what Haag's theorem shows is not that the Hilbert space framework per se is too narrow for describing interacting fields, but rather that a single, universal Hilbert space representation does not suffice for describing both free and interacting fields; ${ }^{33}$ instead, unitarily inequivalent representations of the CCR must be employed.

On any reading, Haag's theorem undermines the interaction picture and the attendant approach to scattering theory. A convincing explanation of why, nevertheless, the interaction picture and perturbation theory work is still lacking, but we indicated one route to such an explanation. Dyson's pessimism is not justified by Haag's theorem because mathematically consistent alternatives to the canonical approach exist. Haag-Ruelle scattering 
theory is available as a mathematically consistent alternative framework for scattering theory. The $\left(\phi^{4}\right)_{1+1}$ model constructed by Glimm and Jaffe is a well-defined Hilbert space representation for this interaction. Since Haag's theorem applies in this case, this example demonstrates that the theorem can be circumvented by adopting a representation that is unitarily inequivalent to the Fock representation for a free field.

The story does not end here. Haag's theorem initiated a pattern that has continued to the present day. Haag's theorem concerns only the infinite volume divergences. Following their success with the $\left(\phi^{4}\right)_{1+1}$ theory, constructive field theorists investigated interactions of different forms and in higher dimensional spaces that are plagued by other types divergences. This work revealed that, in order to obtain well-defined representations, additional assumptions of the Haag's theorem reductio need to be modified or abandoned-for example, the use of the equal-time version of the CCR. The constructive QFT program is far from complete. Critically, a model for a non-trivial interaction in four-dimensional spacetime has yet to be found. ${ }^{34}$ Dyson-esque pessimism is one response to this situation. There is also the by now familiar alternative: speculation that yet further assumptions will have to be abandoned. For example, Klauder (2000) has questioned the assumption of irreducibility.

The absence of discussion of Haag's theorem from the latest generation of textbooks on QFT is an indication that it has dropped out of the consciousness of scholars in the mainstream of the field. Axiomatic and constructive field theorists have digested the moral and moved on to other problems. But this does not mean that Haag's theorem can be ignored by philosophers of science. On the contrary, the theorem provides an entry point into a labyrinth of issues that must be confronted in any satisfactory account of the foundations of QFT.

Acknowledgements: We are grateful to Jon Bain, Gerard Emch, Gordon Fleming, Nick Huggett, Tracy Lupher, Laura Ruetsche, Bert Schroer, and Paul Teller for helpful discussions. Thanks are also due to two anonymous referees for comments that led to substantial improvements. Doreen Fraser thanks the Social Sciences and Humanities Research Council of Canada for financial support in the form of a Doctoral Fellowship.

\section{Appendix: The Algebraic approach to QFT, Weyl algebras, Fock representations, and all that}


A *-algebra is an algebra closed with respect to an involution $\mathcal{A} \ni A \mapsto$ $A^{*} \in \mathcal{A}$ satisfying: $\left(A^{*}\right)^{*}=A,(A+B)^{*}=A^{*}+B^{*},(c A)^{*}=\bar{c} A^{*}$ and $(A B)^{*}=B^{*} A^{*}$ for all $A, B \in \mathcal{A}$ and all complex $c$ (where the overbar denotes the complex conjugate). A $C^{*}$-algebra is a $*$-algebra equipped with a norm, satisfying $\left\|A^{*} A\right\|=\|A\|^{2}$ and $\|A B\| \leq\|A\|\|B\|$ for all $A, B \in \mathcal{A}$, and is complete in the topology induced by that norm.

A representation of a $C^{*}$-algebra $\mathcal{A}$ is a $*$-automorphism $\pi: \mathcal{A} \rightarrow \mathcal{B}(\mathcal{H})$ where $\mathcal{B}(\mathcal{H})$ is the algebra of bounded linear operators on a Hilbert space $\mathcal{H}$. The representation $\pi$ is faithful iff for any $A \in \mathcal{A}, \pi(A)=0$ implies that $A=0 . \pi$ is irreducible iff $\pi(\mathcal{A})^{\prime \prime}=\mathcal{B}(\mathcal{H})$, where $\pi(\mathcal{A})^{\prime \prime}$ is the double commutant of $\pi(\mathcal{A})$ (i.e., the set of all bounded operators that commute with any bounded operator that commutes with $\pi(\mathcal{A}))$. In an irreducible representation every vector $|\psi\rangle \in \mathcal{H}$ is cyclic (i.e., $\{\pi(\mathcal{A})|\psi\rangle\}$ is a dense set). Two representations $\pi$ and $\pi^{\prime}$ are unitarily equivalent iff there is a unitary map $V: \mathcal{B}\left(\mathcal{H}_{\pi}\right) \rightarrow \mathcal{B}\left(\mathcal{H}_{\pi^{\prime}}\right)$ such that $\pi^{\prime}(A)=V \pi(A) V^{-1}$ for all $A \in \mathcal{A}$.

The basic result on representations, called the Gelfand-Naimark-Segal (GNS) theorem, shows that each state (i.e. normed positive linear functional) on the algebra determines a cyclic representation: if $\mathcal{A}$ is a $C^{*}$ algebra and $\omega$ is a state on $\mathcal{A}$, then there is a Hilbert space $\mathcal{H}_{\omega}$, a *automorphism $\pi_{\omega}: \mathcal{A} \rightarrow \mathcal{B}\left(\mathcal{H}_{\omega}\right)$, and a cyclic vector $\left|\Omega_{\omega}\right\rangle \in \mathcal{H}_{\omega}$ such that $\omega(A)=\left\langle\Omega_{\omega}\left|\pi_{\omega}(A)\right| \Omega_{\omega}\right\rangle$ for all $A \in \mathcal{A}$. The GNS representation is the unique (up to unitary equivalence) cyclic representation. A state $\omega$ on a a $C^{*}$-algebra is said to be mixed if it can be written as a nontrivial convex combination of other states; otherwise it is said to be pure. The GNS representation determined by a state $\omega$ is irreducible just in case $\omega$ is pure.

If $\pi: \mathcal{A} \rightarrow \mathcal{B}(\mathcal{H})$ is a representation of the $C^{*}$-algebra $\mathcal{A}$, the associated von Neumann algebra is the weak closure of $\pi(\mathcal{A})$ or, equivalently, the double commutant $\pi(\mathcal{A})^{\prime \prime}$. The folium $\mathfrak{F}(\pi)$ of a representation $\pi$ consists of all states that can be represented as density matrixes on $\mathcal{H}_{\pi}$. The representations $\pi$ and $\pi^{\prime}$ are called disjoint if $\mathfrak{F}(\pi) \cap \mathfrak{F}\left(\pi^{\prime}\right)=\varnothing$. For irreducible representations, disjointness coincides with unitary inequivalence. Two representations $\pi$ and $\pi^{\prime}$ are said to be weakly equivalent if for any state $\omega$ in the folium of $\pi$, any $A_{1}, A_{2}, \ldots, A_{n} \in \mathcal{A}$, for finite $n$, and any positive $\epsilon_{1}, \epsilon_{2}, \ldots, \epsilon_{n}$, there is a state $\omega^{\prime}$ in the folium of $\pi^{\prime}$ such that $\left|\omega\left(A_{i}\right)-\omega^{\prime}\left(A_{i}\right)\right| \leq \epsilon_{i}, i=1,2, \ldots, n$, and vice versa with $\omega$ and $\omega^{\prime}$ interchanged. Fell's theorem guarantees that any two faithful representations of a $C^{*}$-algebra are weakly equivalent. Weyl $C C R$ algebras (to be described below) are $C^{*}$-algebras that encode the CCR in an exponentiated form. Since all representations of a Weyl CCR algebra are 
faithful, it follows from Fell's theorem that they are all weakly equivalent.

A Weyl CCR algebra $\mathcal{W}(\mathcal{T}, \sigma)$ express the CCR on a symplectic space $(\mathcal{T}, \sigma)$ where $\mathcal{T}$ ("test function space") is a vector space and $\sigma$ is a nondegenerate, anti-symmetric, bilinear form on $\mathcal{T}{ }^{35}$ Denoting elements of the quantized space by $\phi$, the "symplectically smeared" fields $\sigma(\phi, f), f \in \mathcal{T}$, are required to satisfy

$$
\left[\sigma\left(\phi, f_{1}\right), \sigma\left(\phi, f_{2}\right)\right]=i \sigma\left(f_{1}, f_{2}\right) I, \quad \text { for all } f_{1}, f_{2} \in \mathcal{T}
$$

In the case of the Klein-Gordon field, $\mathcal{T}$ can be taken to be pairs $(\varphi, \pi)$ of Cauchy data on some time slice, say, $t=0$, consisting of real valued functions in, say, $C_{0}^{\infty}$. The symplectic form is given by $\sigma\left(\varphi_{1}, \pi_{1} ; \varphi_{2}, \pi_{2}\right)=$ $\int\left(\varphi_{1} \pi_{2}-\pi_{1} \varphi_{2}\right) d^{3} x$. Equation (A1) expresses the heuristic form of the CCR

$$
\left[\varphi\left(\mathbf{x}_{1}\right), \varphi\left(\mathbf{x}_{2}\right)\right]=0=\left[\pi\left(\mathbf{x}_{1}\right), \pi\left(\mathbf{x}_{2}\right)\right], \quad\left[\varphi\left(\mathbf{x}_{1}\right), \pi\left(\mathbf{x}_{2}\right)\right]=i \delta\left(\mathbf{x}_{1}, \mathbf{x}_{2}\right) I
$$

Note that, unless otherwise specified, the results stated below hold for arbitrary $(\mathcal{T}, \sigma)$, and not merely for the special case of a linear field theory.

The Weyl algebra $\mathcal{W}(\mathcal{T}, \sigma)$ encodes an exponentiated version of (A1). It is generated by the objects $W(f)$ (which are the formal counterparts of $\exp (-i \sigma(\phi, f)))$ that are required to satisfy

$$
W(f)^{*}=W(-f)
$$

and

$$
W\left(f_{1}\right) W\left(f_{2}\right)=\exp (-i \sigma(\phi, f) / 2) W\left(f_{1}+f_{2}\right)
$$

The algebra is formed by taking complex linear combinations of the $W(f)$. The *-operation comes from (A3) and the requirement that for any element $A$ of the algebra and any $c \in \mathbb{C},(c A)^{*}=\bar{c} A^{*}$. There is a unique norm satisfying $\left\|A^{*} A\right\|=\|A\|^{2}$ for all elements $A$, and taking the closure in this norm produces the promised $C^{*}$-algebra $\mathcal{W}(\mathcal{T}, \sigma)$.

The expectation values

$$
\omega_{\mu}(W(f))=\exp (-\mu(f, f) / 2)
$$

of the generators $W(f)$ determine a state on $\mathcal{W}(\mathcal{T}, \sigma)$ if $\mu$ is a positive symmetric bilinear form on $\mathcal{T}$ obeying the inequality

$$
\left(\sigma\left(f_{1}, f_{2}\right)\right)^{2} \leq 4 \mu\left(f_{1}, f_{1}\right) \mu\left(f_{2}, f_{2}\right), \quad \text { for all } f_{1}, f_{2} \in \mathcal{T}
$$


Such states are called quasi-free. It is worth emphasizing that the Weyl CCR algebra depends only on the real symplectic space $(\mathcal{T}, \sigma)$ and not on the complex structure which needs to be added to form a "one particle Hilbert space" (see below). Roughly speaking, $\sigma$ determines the imaginary part of the Hilbert space inner product while $\mu$ determines the real part. This is one source of unitarily inequivalent representations: the GNS representations of the same $\mathcal{W}(\mathcal{T}, \sigma)$ determined by the states $\omega_{\mu}$ corresponding to different choices of $\mu$ can be unitarily inequivalent.

Kay and Wald (1991, Prop. 3.1) show that for any symplectic space $(\mathcal{T}, \sigma)$ and any $\mu$ satisfying $(\mathrm{A} 6)$, there is an associated "one particle Hilbert space" in the sense that there is a complex Hilbert space $\mathfrak{h}$ and a linear map $K: \mathcal{T} \rightarrow \mathfrak{h}$ such that

(i) $K \mathcal{T}+i K \mathcal{T}$ is dense in $\mathfrak{h}$,

(ii) $\mu\left(f_{1}, f_{2}\right)=\operatorname{real}\left\langle K f_{1}, f_{2}\right\rangle$ for all $f_{1}, f_{2} \in \mathcal{T}$, where $\langle$,$\rangle denotes the inner$ product on $\mathfrak{h}$, and

(iii) $\sigma\left(f_{1}, f_{2}\right)=2 \operatorname{imag}\left\langle K f_{1}, f_{2}\right\rangle$ for all $f_{1}, f_{2} \in \mathcal{T}$.

The symmetric (boson) Fock space $\mathcal{F}(\mathfrak{h})$ over $\mathfrak{h}$ is defined as $\oplus_{n=0}^{n=\infty}\left(\otimes_{s}^{n} \mathfrak{h}^{n}\right)$ where $\mathfrak{h}^{0}=\mathbb{C}$ and $\otimes_{s}^{n} \mathfrak{h}^{n}$ is the symmetrized $n$-fold tensor product of $\mathfrak{h}$ with itself. The creation and annihilation operators define a pair of linear maps

$$
a: f \mapsto a(f), \quad a^{*}: f \mapsto a^{*}(f)
$$

from $\mathfrak{h}$ to $\mathcal{F}(\mathfrak{h})$ such that the $a(f)$ and $a^{*}(f)$ have a common dense domain $D$, and $[a(f), a(g)]=\left[a^{*}(f), a^{*}(g)\right]=0$ and $\left[a(f), a^{*}(g)\right]=\langle f, g\rangle I$ for all $f, g \in \mathfrak{h}$. A representation of the Weyl CCR algebra is said to be a Fock representation just in case

(a) there is a unit vector $|0\rangle \in D$ ("the Fock vacuum") such that $a(f)|0\rangle=0$ for all $f \in \mathfrak{h}$, and

(b) $D$ is the span of $\left\{a^{*}\left(f_{1}\right) a^{*}\left(f_{2}\right) \ldots a^{*}\left(f_{n}\right)|0\rangle\right\}, f_{1}, f_{2}, \ldots f_{n} \in \mathfrak{h}$ and $n=$ $0,1,2 \ldots$.

The GNS representation determined by a pure quasi-free state $\omega_{\mu}$ on $\mathcal{W}(\mathcal{T}, \sigma)$ is a Fock representation. But note that, in accord with what was said above, different pure quasi-free states can produce unitarily inequivalent Fock representations.

In a Fock representation, the total particle number operator $N:=\sum_{\left\{f_{j}\right\}} a^{*}\left(f_{j}\right) a\left(f_{j}\right)$, where the sum is taken over an orthonormal basis $\left\{f_{j}\right\}$ of $\mathfrak{h}$, is a densely defined self-adjoint operator that is independent of the chosen basis, and it has spectrum $\{0,1,2, \ldots\}$. In fact $N$ is characterized 
by the properties that it is self-adjoint with spectrum $\{0,1,2, \ldots\}$ and that it satisfies the commutation relation $N a^{*}(f)=a^{*}(f)(N+1)$ (see Chaiken 1968). A representation of the CCR having such a number operator is necessarily unitarily equivalent up to multiplicity to a Fock representation, i.e. is a direct sum of Fock representations (see Dell'Antonio and Doplicher 1967). 


\section{Notes}

${ }^{1}$ Though, in our view, Bain's explanation is not complete. See Section 6 below.

${ }^{2}$ See Ruetsche (2002) for further discussion of philosophical issues surrounding unitarily inequivalent representations.

${ }^{3}$ See pp. 416 and 419 for sample passages.

${ }^{4}$ Good treatments of Haag's theorem are found in Streater and Wightman (1964), Emch (1972), and Bogulubov et al. (1975), which are devoted to the axiomatic and algebraic approaches to QFT, approaches that are generally ignored by working physicists.

${ }^{5}$ Note, however, that without further restrictions, (1) does not imply (3). The generators of the version of the Weyl CCR algebra given in the Appendix combine the $U$ 's and the $V$ 's.

${ }^{6}$ Note however that the Stone-von Neumann theorem assumes that the configuration space is $\mathbb{R}^{n}$. For systems where this is not the case it is easy to give examples where, although only finitely many degrees of freedom are involved, there are infinitely many unitarily inequivalent representations of the ordinary CCR (1). These niceties will be ignored here.

${ }^{7}$ Here we are following Summers (2001), which can be highly recommended for anyone wishing an overview of history of the Stone-von Neumann theorem, inequivalent representations, and related matters. See also Rosenberg (2004).

${ }^{8}$ Wightman and Schweber credit van Hove as their main inspiration: "Our illustrations ... are based on the fundamental work of van Hove, and our discussion may be regarded as an alternative explication of his results" (1955, p. 825). The footnote to van Hove's (1952) also cites Friedrichs (1953) and "R. Haag, CERN lectures, 1953 (unpublished)."

${ }^{9}$ See Haag (1955, note 15, p. 21).

${ }^{10}$ This does not include the case of zero mass particles. But it will be seen in Section 8 below that this case is "even worse" in that there are unitarily inequivalent representations for the same dynamics.

${ }^{11}$ The classical $\phi^{n}$ field of mass $m$ obeys the equation $\square \phi-m^{2} \phi=\lambda \phi^{n-1}$, where $\lambda$ is the self-coupling constant.

${ }^{12}$ We consider neutral scalar fields for convenience. The theorem also holds in the more general case of fields with spin indices (Streater and Wightman 1964, p. 166). The commutation relations in (4) below are then CCR's or CAR's, as dictated by the Spin-Statistics Theorem. 
${ }^{13}$ Jaffe (1969) writes: "By a general result, called Haag's theorem, the Hamiltonian for [the interacting field] cannot exist on Fock space." (p. 577). And Glimm and Jaffe (1970a) write: "[T]here is a general theorem due to Haag ... which says roughly that in order for these three objects [the physical vacuum, the Hamiltonian for the interacting field, and the Schrödinger dynamics for the interacting field] to exist $\left[\mathcal{H}_{F}\right]$ must be replaced by a new Hilbert space $\left[\mathcal{H}_{R}\right] \ldots "$ (p. 364).

${ }^{14} f$ stands for a "test function." If $\left\{f_{k}\right\}, k=1,2, \ldots$, is a basis for the test function space, then the annihilation operators can be written in the more familiar notation $a_{k}:=a\left(f_{k}\right)$.

${ }^{15}$ The issue of whether Haag's theorem necessarily invokes "strange" or non-Fock representations will be discussed below in Section 7 .

${ }^{16}$ The version of Haag's theorem proved by Streit (1969) and Emch (1972, pp. 247-253) does not make reference to the Poincaré group. This theorem is not contradicted by the mentioend examples of Galilean QFTs since some of the premises of the theorem are not satisfied in these examples (see Fraser (2005) for details.)

${ }^{17}$ Here we are using the standard convention that $\left(\phi^{m}\right)_{n+1}$ is the $\phi^{m}$ field on a spacetime with dimension $n+1$, with $n \geq 1$ being the dimension of space.

${ }^{18}$ We presume that in cases where spacetime smearing is required to produce well-defined operator valued distrubutions it is possible to define a relevant algebra of such smeared fields and that a Haag type moral will apply, i.e. unitarily inequivalent respresentations of the algebra are needed to describe systems with different dynamics. But we know of no precise results to this effect.

${ }^{19}$ See Bjorken and Drell (1965, Chapters 16, 17) or Schweber (1962, Chapters 11,13$)$.

${ }^{20}$ Actually, things are a bit more complicated because renormalization affects the commutation relations. The renormalized interaction picture field $\phi_{\text {ren }}$ obeys

$$
\left[\phi_{r e n}(\mathbf{x}, t), \frac{\partial \phi_{r e n}(\mathbf{y}, t)}{\partial t}\right]=\frac{i}{Z} \delta(\mathbf{x}-\mathbf{y}) \text { where } Z=0
$$

But, strictly speaking, the 'renormalized' interaction picture representation for $\phi_{\text {ren }}(\mathbf{x}, t)$ cannot be employed to evaluate scattering matrix elements. To do this, it is essential that the free field $\phi_{I}(\mathbf{x}, t)$ be represented on its Fock 
space, which contains $n$-particle states. $\phi_{\text {ren }}(\mathbf{x}, t)$, however, cannot be given a Fock space representation in the standard way because it obeys the above non-standard ETCCR's.

${ }^{21}$ The Glimm and Jaffe construction of the renormalized Hilbert space $\mathcal{H}_{R}$ for $\left(\phi^{4}\right)_{1+1}$ field uses the algebraic approach sketched in the Appendix. They produce a representation $\pi$ of the algebra $\mathcal{A}$ which is the norm closure of $\cup_{B} \mathcal{A}(B)$, where $\mathcal{A}(B)$ is the von Neumann algebra generated by bounded functions of the smeared field $\phi(f, 0)=\int \phi(\mathbf{x}, 0) f(x) d x$ with $f$ having support in a bounded region $B$ of space. They prove that for each bounded region $B$ there is a unitary map $U_{B}$ from the Hilbert space in which the representation of the free field lives to $\mathcal{H}_{R}$ such that $\pi(A)=A_{R}=U_{B} A U_{B}^{*}$ for all $A \in \mathcal{A}$.

${ }^{22}$ One could seek to control ultraviolet divergences by using a preferred set of states. In the case of the free scalar field the preferred states-called Hadamard states-are those that yield a well-defined expectation value for the renormalized stress-energy tensor of the field (see Wald 1994). Any two quasi-free Hadamard states determine representations of a Weyl CCR algebra that are locally quasi-equivalent, i.e. any subrepresentation of one is unitarily equivalent to some subrepresentation of the other (see Verch 1994). Perhaps there is some preferred set of states for interacting fields which, in analogy with Hadamard states, control the far-ultraviolet modes of the field and which underwrite a result to the effect that any such state determines a representation of the Weyl CCR algebra that is locally quasi-equivalent to the representation determined by a quasi-free Hadamard state.

${ }^{23}$ Bain (2000) argues that Haag's theorem can be evaded by employing the LSZ asymptotic condition, and attributes its success to the fact that it uses a weak limit (pp. 384-6). The LSZ asymptotic condition is a special case of Haag-Ruelle scattering theory. Thus, while it is true that the LSZ asymptotic condition provides a formulation of scattering theory that is consistent with Haag's theorem, it is misleading to attribute its success to the fact that it employs a weak limit since Haag-Ruelle scattering theory demonstrates that the $S$-matrix can also be consistently defined using strong limits.

${ }^{24}$ Koshmanenko $(1978,1979)$ develops a generalized version of scattering theory with different state spaces and shows that Haag-Ruelle scattering theory is a special case.

${ }^{25}$ See also Streater (1975, pp. 797-8).

${ }^{26}$ This claim is explicitly made in some discussions. Lopuszanski's (1961) 
version of Haag's theorem is said to imply the need to use a "myriotic representative of the commutation relations" (p. 747). "Myriotic representation" is another name for non-Fock representation; see below.

${ }^{27}$ This example is used over and over in the literature. E.g. Wightman and Schweber (1955, pp. 824-5) use it to illustrate the moral that the choice of representation depends on the dynamics has the same structure: just make the $\alpha_{k}$ functions of the coupling constant describing the interaction.

${ }^{28}$ Contrast the views of Teller (1995) and Wald (1994).

${ }^{29}$ See Summers (2001) and Kronz and Lupher (2003).

${ }^{30}$ Streater and Wightman write that for systems with an infinite number of degrees of freedom "the kinematics gets mixed up with the dynamics in the sense that the dynamics determine which representation of the canonical commutation relations we must use" (1964, p. 168).

${ }^{31}$ See Earman (2004) for a user friendly introduction to some of the foundational issues raised by spontaneous symmetry breaking.

${ }^{32}$ In the Wightman approach to QFT, the Wightman reconstruction theorem (Streater and Wightman 1964, Sec. 3-4) shows that if the Wightman functions possess certain properties, then there is a separable Hilbert space and a unique Poincaré invariant state-the vacuum-such that the Wightman functions are equal to the $n$-point functions of this state; and further, any other representation with these vacuum expectation values is unitarily equivalent to this one. From the algebraic point of view, the Wightman field operators form a Borchers algebra (see Borchers 1962), and the axioms of Wightman field theory imply that the Wightman functions define a positive linear functional on this algebra, so that the GNS theorem can be applied.

${ }^{33}$ Or even for describing free fields of different masses.

${ }^{34}$ For a report on the state of constructive QFT as of 2000, see Rivasseau (2000).

${ }^{35}$ The treatment given below closely follows that of Kay and Wald (1991) and Wald (1994), to which the reader is referred for details. 


\section{References}

Arageorgis, A., Earman, J., and Ruetsche, L.: 2002, 'Weyling the Time Away: The Non-Unitary Implementability of Quantum Field Dynamics on Curved Spacetime', Studies in the History and Philosophy of Modern Physics 33: $151-184$.

Bain, J.: 2000, 'Against particle/field duality: asymptotic particle states and interpolating fields in interacting QFT (Or: Who's afraid of Haag's theorem?)', Erkenntnis 53: 375-406.

Barton, G.: 1963, Introduction to advanced field theory, Interscience Publishers, New York.

Baumann, K.: 1987, 'On relativistic irreducible quantum fields fulfilling the CCR', Journal of Mathematical Physics 28: 697-704.

Baumann, K.: 1988, 'On canonical irreducible quantum field theories describing bosons and fermions', Journal of Mathematical Physics 29: 1225-1230.

Bjorken, J. D. and Drell, S. D.: 1965, Relativistic quantum fields, McGrawHill, New York.

Bogoliubov, N. N., Logunov, A. A., and Todorov, I. T.: 1975, Introduction to axiomatic quantum field theory, W. A. Benjamin, Reading, MA.

Borchers, H.-J.: 1962, 'On structure of the algebra of operators', Nuovo Cimento 24: 214-236.

Chaiken, J. M.: 1968, 'Number operators for representations of the canonical commutation relations', Communications in Mathematical Physics 8: 164184.

Dell'Antonio, G. F. and Doplicher, S.: 1967, 'Total number of particles and Fock representation', Journal of Mathematical Physics 8: 663-666.

Earman, J.: 2004, 'Curie's Principle and Spontaneous Symmetry Breaking', to appear in International Studies in Philosophy of Science 18: 173-198.

Emch, G.: 1972, Algebraic methods in statistical mechanics and quantum field theory, Wiley-Interscience, New York.

Florig, M., and Summers, S. J.: 2000, 'Further representations of the canonical commutation relations,' Proceedings of the London Mathematical Society 80: $451-490$. 
Fraser, D.: 2005, Philosophical implications of the treatment of interactions in QFT, PhD thesis, University of Pittsburgh.

Friedrichs, K.: 1953, Mathematical aspects of the quantum theory of fields, Interscience, New York.

Gårding, L. and Wightman, A. S.: 1954, 'Representations of the commutation relations', Proceedings of the National Academy of Sciences 40: 622-626.

Ginibre, J. and Velo, G.: 1970, 'Renormalization of a quadratic interaction in the Hamiltonian formalism', Communications in Mathematical Physics 18: 65-81.

Glimm, J. and Jaffe, A.: 1968, 'A $\lambda \phi^{4}$ field theory without cutoffs. I', Physical Review 176: 1945-1951.

Glimm, J. and Jaffe, A.: 1970a, 'The $\lambda\left(\phi^{4}\right)_{2}$ quantum field theory without cutoffs: II. The field operators and the approximate vacuum', Annals of Mathematics 91: 362-401.

Glimm, J. and Jaffe, A.: 1970b, 'The $\lambda\left(\phi^{4}\right)_{2}$ quantum field theory without cutoffs: III. The physical vacuum', Acta Mathematica 125: 203-267.

Greenberg, O. W.: 1959, 'Haag's theorem and clothed operators', Physical Review 115: 706-710.

Greenberg, O. W. and Licht, A. L.: 1963, 'Quantum field-theory model whose truncated vacuum expectation values vanish yeyond some order', Journal of Mathematical Physics 4: 613-614.

Haag, R.: 1955, 'On quantum field theories', Det Kongelige Danske Videnskabernes Selskab, Matematisk-fysiske Meddelelser 29, nr. 12: 1-37.

Haag, R.: 1958, 'Quantum field theories with composite particles and asymptotic conditions', Physical Review 112: 669-673.

Hall, D. and Wightman, A. S.: 1957, 'A theorem on invariant analytic functions with applications to relativistic quantum field theory', Det Kongelige Danske Videnskabernes Selskab, Matematisk-fysiske Meddelelser 31, nr. 5: 1-41.

Heathcote, A., 1989, 'Theory of causality: causality = interaction (as defined by a suitable quantum field theory)', Erkenntnis 31: 77-108.

Hepp, K.: 1969, Théorie de la renormalisation, Lecture Notes in Physics, Vol. 2, Springer-Verlag, Berlin. 
Huggett, N. and Weingard, R.: 1994, 'Interpretations of quantum field theory', Philosophy of Science 61: 370-388.

Jaffe, A.: 1969, 'Whither axiomatic field theory?', Reviews of Modern Physics 41: $576-580$.

Jaffe, A.: 1999, 'Where does quantum field theory fit into the big picture?', in T. Y. Cao (ed.), Conceptual foundations of quantum field theory, Cambridge University Press, New York, pp. 136-147.

Jost, R.: 1961, 'Properties of Wightman functions', in E. R. Caianiello (ed.), Lectures on field theory and the many-body problem, Academic Press, New York, pp. 127-145.

Kay, B.: 1978, 'Linear spin-zero quantum fields in external gravitational and scalar fields. I. A one particle structure for the stationary case', Communications in Mathematical Physics 62: 55-70.

Kay, B. and Wald, R. M.: 1991, 'Theorems and the Uniqueness and Thermal Properties of Stationary, Nonsingular, Quasifree States on Spacetimes with a Bifurcate Killing Horizon', Physics Reports 207: 49-136.

Klauder, J. R.: 2000, Beyond conventional quantization, Cambridge University Press, Cambridge.

Koshmanenko, V. D.: 1978, 'Scattering theory with different state spaces of perturbed and free system', Reports on Mathematical Physics 14: 185-206.

Koshmanenko, V. D.: 1979, 'Haag-Ruelle scattering theory as scattering theory in different spaces', Theoretical and Mathematical Physics 38: 109119 .

Kronz, F. and Lupher, T.: 2003, 'Unitarily inequivalent representations in algebraic quantum theory', pre-print.

Lévy-Leblond, J.-M.: 1967, 'Galilean quantum field theories and a ghostless Lee model', Communications in Mathematical Physics 4: 157-176.

Lévy-Leblond, J.-M.: 1971, 'Galilei group and Galilean invariance', in E. M. Loebl (ed.), Group theory and its applications, Vol II, Academic Press, New York, pp. 222-299.

Lopuzanski, J.: 1961, 'A criterion for the free character of fields', Journal of Mathematical Physics 2: 743-747. 
Lopuzanski, J.: 1965, 'On the unitary inequivalent representations in the quantum field theory and the many body problem', Acta Physica Hungarica 19: 29-44.

Manceau, J. and Verbeure, A. : 1968, 'Quasi-Free States of the C.C.R.Algebra and Bogoliubov Transformations', Communications in Mathematical Physics 9, 293-302.

Peskin, M. E. and Schroder, D. V.: 1995, An introduction to quantum field theory, Addison-Wesley, Reading, MA.

Pohlmeyer, K.: 1969, 'The Jost-Schroer theorem for zero-mass fields', Communications in Mathematical Physics 12: 204-211.

Powers, R. T.: 1967, 'Absence of interaction as a consequence of good untraviolet behavior in the case of a local Fermi field', Communications in Mathematical Physics 4: 145-156.

Prugovečki, E.: 1981, Quantum mechanics in Hilbert space, 2nd ed., Academic Press, New York.

Reed, M. and Simon, B.: 1975, Methods of modern mathematical physics, Vol. II, Fourier analysis, self-adjointness, Academic Press, New York.

Reed, M. and Simon, B.: 1979, Methods of modern mathematical physics, Vol. III, Scattering theory, Academic Press, New York.

Rivasseau, V.: 2000, 'Constructive field theory and applications: Perspectives and open problems', Journal of mathematical Physics 41: 3764-3775.

Roman, P.: 1969, Introduction to quantum field theory, John Wiley and Sons, New York.

Rosenberg, J.: 2004, 'A Selective History of the Stone-von Neumann Theorem', Contemporary Mathematics 365: 331-353.

Ruelle, D.: 1962, 'On the asymptotic condition in quantum field theory', Helvetica Physica Acta 35: 147-163.

Ruetsche, L.: 2002, 'Interpreting quantum field theory', Philosophy of Science 69: 348-378.

Schweber, S. S.: 1962, An introduction to relativistic quantum field theory, Harper and Rowe, New York.

Sklar, L.: 2000, Theory and truth, Oxford University Press, New York. 
Streater, R. F.: 1975, 'Outline of axiomatic relativistic quantum field theory', Reports on Progress in Physics 38: 771-846.

Streater, R. F. and Wightman, A. S.: 1964, PCT, spin and statistics, and all that, W. A. Benjamin, New York.

Sterman, G.: 1993, An introduction to quantum field theory, Cambridge University Press, Cambridge.

Streit, L.: 1969, 'A generalization of Haag's theorem', Nuovo Cimento LXIIA, N. 3: 673- 680 .

Summers, S. J.: 2001, 'On the Stone-von Neumann uniqueness theorem and its ramifications', in M. Rédei and M. Stöltzner (eds), John von Neumann and the foundations of quantum physics, Kluwer Academic, Dordrecht, pp. $135-152$.

Teller, P.: 1995, An interpretative introduction to quantum field theory, Princeton University Press, Princeton, NJ.

Ticciati, R.: 1999, Quantum field theory for mathematicians, Cambridge University Press, Cambridge, UK.

van Hove, L.: 1952, 'Les difficultés de divergences pour un modèle particulier de champ quantifié,' Physica 18, no 3: 145-159.

Verch, R.: 1994, 'Local definiteness, primitivity, and quasiequivalence of quasi-free Hadamard quantum states in curved spacetime', Communications in Mathematical Physics 160: 507-536.

Wald, R. M.: 1994, Quantum field theory on curved spacetime and black hole thermodynamics, University of Chicago Press, Chicago.

Weinberg, S.: 1995, The quantum theory of fields, Vol. I, Foundations, Cambridge University Press, Cambridge, UK.

Weinberg, S.: 1996, The quantum theory of fields, Vol. II, Modern applications, Cambridge University Press, Cambridge, UK.

Weinless, M.: 1969, 'Existence and Uniqueness of the Vacuum for Linear Quantized Fields', Journal of Functional Analysis 4: 350-379.

Wightman, A. S.: 1967, 'Introduction to some aspects of the relativistic dynamics of quantized fields," in M. Lévy (ed.), Cargèse lectures in theoretical physics, High energy electromagnetic interactions and field theory, Gordon and Breach, New York, pp. 171-291. 
Wightman, A. S. and Schweber, S.: 1955, 'Configuration space methods in relativistic quantum field theory. I', Physical Review 98: 812-837. 